\title{
A FAMILY OF ASYMPTOTICALLY STABLE CONTROL LAWS FOR FLEXIBLE ROBOTS BASED ON A PASSIVITY APPROACH
}

\author{
NAGW-1333 \\ by
}

Leonardo Lanari and John T. Wen

\author{
Rensselaer Polytechnic Institute \\ Electrical, Computer, and Systems Engineering \\ Troy, New York 12180-3590
}

February 1991

CIRSSE REPORT \#85 

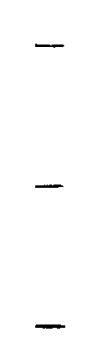

-

$+$

-

-

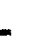




\title{
A family of asymptotically stable control laws for flexible robots based on a passivity approach
}

\author{
Leonardo Lanari and John T. Wen \\ Dipartimento di Informatica e Sistemistica \\ Università di Roma 'La Sapienza', Via Eudossiana 18, 00184 Roma, Italy \\ NASA Center for Intelligent Robotic Systems for Space Exploration \\ Rensselaer Polytechnic Institute, Troy, NY 12180-3590, USA
}

July 11, 1991

\begin{abstract}
A general family of asymptotically stabilizing control laws is introduced for a class of nonlinear Hamiltonian systems. The inherent passivity property of this class of systems and the Passivity Theorem are used to show the closed-loop input/output stability which is then related to the internal state space stability through the stabilizability and detectabilty condition. Applications of these results include fully actuated robots, flexibe joint robots, and robots with link flexibility.
\end{abstract}

\section{Keywords}

Passivity; flexible manipulator; flexible beam; flexible joint; Lyapunov analysis.

\section{Introduction}

Inherent passivity in fully actuated mechanical systems has long been recognized and exploited for their stabilization $[1,2]$. However, when some degrees of freedom are not directly actuated, for example, in flexible robots, the passivity property becomes less useful as damping can only be directly added in certain subspace.

In this paper, we present a family of asymptotically stable set point and tracking controllers for a class of mechanical systems. The approach is applicable even when the openloop system has no inherent damping. The results are then applied to some common models of flexible joint robots and flexible beams. 
The basic controller structure is the sum of a model-based feedforward and a modelindependent feedback. The feedforward is chosen to form an error equation so that the ensuing steps for feedback stabilization can be applied:

- Choose a static feedback so that the system is passive, stabilizable and detectable with respect to a particular input/output pair (usually corresponding to the force/torque inputs and velocity measurements of the degrees of freedom that are actuated).

- Choose a strictly passive feedback between the passive input/output pair. From the Passivity Theorem, the closed-loop system is input/output stable.

- If the feedback is chosen to preserve stabilizability and detectability, the internal states are also asymptotically stable.

Passivity property for flexible jointed robot was recognized in [3] and indeed was used in a PD-type controller design. The method requires inherent damping in both joints and motors. Similar results without requiring the inherent damping has recently appeared in [4]. The result on PD stabilization of flexible beams was first shown in [5]. Our approach was independently conceived and contains a number of unique features as summarized below:

1. Both flexible joint robots and flexible beam are considered as special cases of a more general framework of under-actuated Hamiltonian systems with elastic coupling between actuated and un-actuated degrees of freedom.

2. Any passive feedback is allowed without affecting stability. Examples have shown that higher order feedback (in contrast to the usual proportional-derivative (PD)) can lead significant improved performance.

3. Generalization to tracking control has been addressed from various perspectives.

There has been many published work on applying exact linearization for the control of flexible robots, for a summary see [6]. In general, this approach requires the exact model information, linear spring assumption, and zero gyroscopic force coupling. Furthermore, the feedforward compensation (for linearization) and the feedback stabilization are intertwined and errors in the feedforward may affect the closed loop stability in an adverse way. In contrast, our approach requires much less model information in the set point control case (only the spring characteristics is needed), can be extended to the nonlinear spring case and fully coupled dynamic model, and the additive separation between the feedback and feedforward implies that error in feedforward does not lead to instability. The price to pay is that the closed loop performance cannot be arbitrarily assigned.

For flexible beams (and more generally, flexible structures), the importance of passivity was well recognized in [7]. But only the set point control was considered and the uniform damping for flexible modes was assumed. There was also some work involving open-loop, computed torque type of control $[8,9]$. We drew from this work for the construction of the feedforward trajectory for the error system. A general passive controller design for fully actuated arms was proposed in [10] which is later applied to a multiple-flexible-link robot in [11]. The method here is similar to this approach, but no inherent damping is required as in [11]. 
The rest of the paper is organized as follows. Some background results that will be used in the stability analysis are collected in Section 2. The main procedure is outlined in Section 3. Three application examples, flexible joint robots, flexible beam, and fully actuated robot, are then considered in Sections 4, 5, 6, respectively. Generalization to the trajectory tracking problem is considered in Section 7. Simulation results involving a single flexible joint robot are summarized in Section 9.

\section{Preliminaries}

The time evolution of 'energy' is an important and useful characterization of stability for physical systems, linear and nonlinear alike. Energy based stability analysis has been widely applied to the study of systems such as electrical networks, mechanical structures, thermal systems, etc [12]. The concept of passivity is traditionally defined as an input/output (I/O) condition [13] describing a common class of physical systems which do not generate energy. Relationship between I/O passivity and state space parameters was extensively explored in the 60's [14] in part by using the Lyapunov's method. In this section, we will summarize some basic definition and results that will be useful for the rest of the paper.

Define the input and output signal spaces, $U_{e}, \mathcal{Y}_{e}$, respectively, as extended spaces $L_{2 e}\left(\mathbf{R}_{+}, \mathbf{R}^{m}\right)$. Let $P_{T}$ denote the operator which truncates a signal at time $T$. Define the truncated inner product by

$$
\langle u(\cdot), v(\cdot)\rangle_{T} \triangleq\left\langle P_{T} u(\cdot), P_{T} v(.)\right\rangle_{2}=\int_{0}^{\infty}\left(P_{T} u(t)\right)^{T} P_{T} v(t) d t .
$$

By a dynamical system, we mean an $1 / O$ mapping $H: \mathcal{U}_{e} \rightarrow \mathcal{Y}_{e}$. The input-output stability considered here is the finite-gain $\mathrm{I} / \mathrm{O}$ stability. A system $H$ is said to be finite-gain $\mathrm{I} / \mathrm{O}$ stable if there exists a constant $k$ such that

$$
\left\|P_{T} y\right\| \leq k\left\|P_{T} u\right\| \quad \text { for all } T \geq 0 .
$$

$H$ is passive if

$$
\langle y, u\rangle_{T} \geq 0 \quad \text { for all } T \geq 0 .
$$

The concept of passivity can be generalized to dissipativity [15]. A system $H$ is dissipative with respect to the triple $(Q, S, R)$ if

$$
\langle y, Q y\rangle_{T}+2\langle y, S u\rangle_{T}+\langle u, R u\rangle_{T} \geq 0
$$

for all $T \geq 0$ and $u \in \mathcal{U}_{e}$, where $Q, S$ and $R$ are memoryless bounded operators with $Q$ and $R$ self-adjoint. Clearly, a finite-gain stable system is dissipative with respect to $\left(-I, 0, k^{2} I\right)$, while a passive system is dissipative with respect to $\left(0, \frac{1}{2} I, 0\right)$.

An important theorem which can be used to determine the I/O stability of the interconnection of passive systems is the Passivity Theorem. In its simplest form, it states that if the open-loop system is passive and the feedback system is strictly passive, then the closed-loop system is $L_{2}$-stable i.e. finite-gain $\mathrm{I} / \mathrm{O}$ stable.

$\mathrm{I} / \mathrm{O}$ stability infers internal state space asymptotic stability if the closed-loop system is stabilizable and zero-state detectable (if these properties hold globally, the internal stability 
is also global). A system $H$ is said to be zero-state detectable if $u(t) \equiv 0$ and $y(t) \rightarrow 0$ imply that the state $x(t) \rightarrow 0$. For linear systems, this corresponds to detectability. Under the stabilizability and detectability conditions, a dissipative system with $Q<0$, i.e. a finite gain I/O stable system, has an asymptotically stable equilibrium at zero.

\section{Main procedure}

The class of systems considered in this paper is described by the following dynamical equation of motion:

$$
M(\theta) \ddot{\theta}+D(\dot{\theta})+C(\theta, \dot{\theta}) \dot{\theta}+f(\theta)=B u
$$

where $\theta \in \mathbf{R}^{n}$ is the displacement vector, $u \in \mathbf{R}^{m}$ is the input force vector, $M$ is the mass-inertia matrix, $D$ is the viscous damping and Coulomb friction, $C$ corresponds to the centrifugal and coriolis forces, and $f$ contains the gravity force, spring coupling force, etc. Note that for space applications, the gravity force can be ignored.

Most mechanical systems belong to this class; additional assumptions will be imposed later as required. Particular systems of interest that can be considered include fully actuated robots, flexible joint robots, robots with flexible links, and satellites with flexible appendages.

Fit For the general discussion, we assume zero damping, i.e., $D(\dot{\theta})=0$. All the results are of course valid for the damped case also.

We will first consider the output set point control problem.

Assume the measured outputs are $B^{T} \theta$ and $B^{T} \dot{\theta}$, i.e., the generalized coordinate and velocity that are directly actuated. Suppose the output of interest is

$$
y=C \theta \text {. }
$$

Choose a feedback control law $u$ based on the measured output, so that $y(t)$ asymptotically converges to the desired output set point $y_{d e s}$.

Based on the inherent passivity property of this class of systems, the general procedure described below can be used to construct a solution to the output set point control problem. Extension to output tracking will be addressed in Section 7 .

1. Steady State Analysis. The first step is to find a desired state $\theta_{\text {des }}$ and a feedforward $u_{\text {ff }}$ such that

$$
\begin{aligned}
C \theta_{\text {des }} & =y_{\text {des }} \\
B u_{f f} & =f\left(\theta_{\text {des }}\right) .
\end{aligned}
$$

If these equations are solvable, then the feedforward control can be used to form the error system:

$$
M(\theta) \ddot{\theta}+D(\dot{\theta})+C(\theta, \dot{\theta}) \dot{\theta}+f(\dot{\theta})-f\left(\theta_{\text {des }}\right)=B u_{0}
$$

where $u=u_{0}+u_{f f}$. 
2. Error system Stabilization. Assume that with a static feedback $u_{0}=g\left(B^{T} \theta\right)$, the map from $u_{0}$ to $B^{T} \dot{\theta}$ is passive (this assumption will be justified for a number of applications). Then any strictly passive map from $B^{T} \dot{\theta}$ to $u_{0}$ can be used to feedback $\mathrm{I} / \mathrm{O}$ stabilize the error system. If the closed loop system is further stabilizable and zero-state detectable with respect to $u_{o}$ and $B^{T} \dot{\theta}$, respectively, then the zero error state is asymptotically stable.

We will focus on three examples, flexible joint robots, flexible beams, and fully actuated robots, to demonstrate the application of the above simple approach.

\section{Remarks:}

1. If the system is linear, then the passivity of the original system (between $u$ and $B^{T} \dot{\theta}$ ) implies the passivity of the error system (between $u_{o}$ and $B^{T} \dot{\theta}$ ). For nonlinear systems, additional assumption on $f$ needs to be placed, for example, the joint stiffness is sufficiently strong relative to the gravity load for flexible joint robots.

2. It is well known that passive linear systems are necessarily minimum phase and, conversely, a minimum phase plant can be rendered passive through a static state feedback. A similar relationship for nonlinear systems has also been recently published [16]. It is shown that a nonlinear system can be rendered passive by static feedback (i.e., it is feedback equivalent to a passive system) if and only if the zero-dynamics are weaklyminimum phase and the relative degree is one. It is known that flexible joint robots and flexible beams have stable zero-dynamics with respect to the motor velocity and hub velocity, respectively. We will show that the static position feedback renders these systems passive.

3. The classical proportional-derivative (PD) control law (for the actuated variable) is a special case of the family of control laws developed here. However, the velocity feedback can be augmented by any passive system in parallel. Through an example, we will see that the dynamic nature of the passive system can be exploited to enhance the closed-loop performance.

4. As it will be shown in the application examples in sections to follow, the above analysis does not require any structural damping in the model. Damping, however, will be useful in the output tracking problem.

\section{Application to Flexible Joint Robots}

Consider the general model for an $n$-link flexible joint robot ( $2 n$ degrees of freedom) [1i]. This model contains the gyroscopic forces that are commonly assumed approximately zero $[18,19,20]$. Denote the link angle vector by $\theta_{\ell}$ and motor angle vector by $\theta_{m}$. Define $\theta=\left[\begin{array}{ll}\theta_{\ell}{ }^{T} & \theta_{m}{ }^{T}\end{array}\right]^{T}$. The dynamic equation of motion is given by

$$
M(\theta) \dot{\theta}+C(\theta, \dot{\theta}) \dot{\theta}+g(\theta)+k(\theta)=B u
$$


where $B$ is of the form $\left[\begin{array}{ll}0 & I\end{array}\right]^{T}$ due to the assumption that only motor shafts are actuated, $g(\theta)$ denotes the gravity load, and $k(\theta)$ denotes the spring coupling between motor shafts and the link shafts.

\subsection{Feedforward Compensation based on Steady State Analysis}

The control objective is to steer $\theta_{\ell}$ to some desired constant $\theta_{\ell_{\text {des }}}$ (i.e., in (2), $y=\theta_{\ell}$ ). The first step is to form an error system:

$$
M(\theta) \ddot{\theta}+C(\theta, \dot{\theta}) \dot{\theta}+g(\theta)-g\left(\theta_{\text {des }}\right)+k(\theta)-k\left(\theta_{\text {des }}\right)=B u-g\left(\theta_{\text {des }}\right)-k\left(\theta_{\text {des }}\right) .
$$

In order to cancel the additional terms on the right hand side, we need to find a feedforward torque $u_{f f}$ and a desired set of angles $\theta_{\text {des }}$ (as in (3)-(4)) that satisfy

$$
\begin{aligned}
u & =u_{o}+u_{f f} \\
B u_{f f} & =g\left(\theta_{\text {des }}\right)+k\left(\theta_{\text {des }}\right) .
\end{aligned}
$$

Equation (8) can be restated as follows: for a given $\theta_{\ell_{d e s}}$, find $\theta_{m_{\text {des }}}$ and $u_{f f}$ such that

$$
\begin{aligned}
0 & =\tilde{B}\left(g\left(\theta_{\text {des }}\right)+k\left(\theta_{\text {des }}\right)\right) \\
u_{f f} & =\left(B^{T} B\right)^{-1} B^{T}\left(g\left(\theta_{\text {des }}\right)+k\left(\theta_{\text {des }}\right)\right)=B^{T}\left(g\left(\theta_{\text {des }}\right)+k\left(\theta_{\text {des }}\right)\right)
\end{aligned}
$$

where $\widetilde{B}$ is the annihilator matrix for $B$, i.e., $\widetilde{B} B=0$ or $\widetilde{B}=\left[\begin{array}{ll}I & 0\end{array}\right]$. The terms $g\left(\theta_{\text {des }}\right)$ and $k\left(\theta_{\text {des }}\right)$ are usually in the form

$$
\begin{aligned}
& g\left(\theta_{\text {des }}\right)=\left[\begin{array}{c}
g_{1}\left(\theta_{\ell_{\text {des }}}\right) \\
0
\end{array}\right] \\
& k\left(\theta_{\text {des }}\right)=\left[\begin{array}{c}
N k_{1}\left(\theta_{\ell_{\text {des }}}, \theta_{m_{\text {des }}}\right) \\
-k_{1}\left(\theta_{\ell_{\text {des }}}, \theta_{m_{\text {des }}}\right)
\end{array}\right] .
\end{aligned}
$$

This implies that $u_{f f}$ and $\theta_{m_{\text {des }}}$ should satisfy

$$
\begin{aligned}
u_{f f} & =\frac{g_{1}\left(\theta_{\ell_{\text {des }}}\right)}{N} \\
k_{1}\left(\theta_{\ell_{\text {des }}}, \theta_{m_{\text {des }}}\right) & =-\frac{g_{1}\left(\theta_{\ell_{\text {des }}}\right)}{N} .
\end{aligned}
$$

To solve (14), we assume that for a given $\theta_{\ell_{\text {des }}}, \nabla_{\theta_{m}} k_{1}\left(\theta_{\ell_{d e s}}, \theta_{m}\right)$ is invertible in some open set in $\theta_{m}$. Then by the Implicit Function Theorem [21], there exists a locally unique solution $\theta_{m_{\text {des }}}$ to the equation (14). A common form of $k_{1}$ is

$$
\left(k_{1}\right)_{i}\left(\theta_{\ell}, \theta_{m}\right)=f\left(N_{i} \theta_{\ell i}-\theta_{m i}\right)
$$

where $N_{i}$ is the gear ratio of the $i$ th joint and $f$ is monotonically increasing, continuously differentiable, and the range of $f$ is $\mathbf{R}$. In that case, since $f$ is globally invertible, a unique solution, $\theta_{m_{d e s}}$, to (14) can be found for any $\theta_{\ell_{\text {des }}}$. 


\subsubsection{Passivity}

With the desired motor angle and feedforward torque chosen as in (13) and (14), the equation of motion becomes

$$
M(\theta) \ddot{\theta}+C(\theta, \dot{\theta}) \dot{\theta}+g(\theta)-g\left(\theta_{\text {des }}\right)+k(\theta)-k\left(\theta_{\text {des }}\right)=B u_{o} .
$$

If the sum of the gravitational potential energy and spring potential energy is positive semidefinite in $\Delta \theta, \Delta \theta=\theta-\theta_{\text {des }}$, then one can show that the map from $u_{o}$ to $\dot{\theta}$ is passive. This is not true in general since $\theta_{\ell_{\text {dee }}}$ can be arbitrarily chosen. Therefore, we introduce an artificial potential energy by using a proportional feedback; more specifically, choose $u_{o}$ to be

$$
u_{o}=u_{1}-K_{p} B^{T} \Delta \theta
$$

Assume that it is possible to choose $K_{p}$ so that for some $\delta>0$

$$
\nabla_{\theta} g\left(\theta_{\text {des }}\right)+\nabla_{\theta} k\left(\theta_{\text {des }}\right)+B K_{p} B^{T} \geq \delta I>0 .
$$

Consider $g(\theta)$ and $k(\theta)$ as modeled by (11) and (12), respectively, where $k_{1}$ is

$$
k_{1}\left(\theta_{\ell}, \theta_{m}\right)=f\left(N \theta_{\ell}-\theta_{m}\right)
$$

$f$ is a monotonically increasing function and $N$ is a diagonal matrix containing the gear ratios. Then condition (17) becomes

$$
\therefore\left[\begin{array}{cc}
\nabla_{\theta_{\ell}} g_{1}\left(\theta_{\ell_{\text {des }}}\right) & 0 \\
0 & 0
\end{array}\right]+\left[\begin{array}{cc}
N^{2} \nabla f\left(N \theta_{\ell_{\text {dee }}}-\theta_{m_{\text {des }}}\right) & -N \nabla f\left(N \theta_{\ell_{\text {des }}}-\theta_{m_{\text {dess }}}\right) \\
-N \nabla f\left(N \theta_{\ell_{\text {des }}}-\theta_{m_{\text {des }}}\right) & \nabla f\left(N \theta_{\ell_{\text {des }}}-\theta_{m_{\text {des }}}\right)
\end{array}\right]+\left[\begin{array}{cc}
0 & 0 \\
0 & K_{p}
\end{array}\right]>0
$$

If the spring at the joint is sufficiently stiff in the sense that

$$
N^{2} \nabla f\left(N \theta_{\ell_{\text {des }}}-\theta_{m_{\text {des }}}\right)>-\nabla_{\theta_{\ell}} g_{1}\left(\theta_{\ell_{\text {des }}}\right)
$$

then condition (19) is satisfied for a sufficiently large $K_{p}$.

Now consider the following scalar function

$$
V(\Delta \theta, \dot{\theta})=\frac{1}{2} \dot{\theta}^{T} M(\theta) \dot{\theta}+U(\Delta \theta)
$$

where the first term on the right hand side is the kinetic energy and the second term is the sum of the potential energies:

$$
\begin{gathered}
U(\Delta \theta)=G\left(\Delta \theta+\theta_{\text {des }}\right)-G\left(\theta_{\text {des }}\right)-g\left(\theta_{\text {des }}\right)^{T} \Delta \theta+K\left(\Delta \theta+\theta_{\text {des }}\right)-K\left(\theta_{\text {des }}\right) \\
-k\left(\theta_{\text {des }}\right)^{T} \Delta \theta+\frac{1}{2} \Delta \theta^{T} B K_{p} B^{T} \Delta \theta
\end{gathered}
$$

where $G$ and $K$ are the gravitational and spring potential energies, respectively. Under the assumption that (17) is satisfied, $U(\Delta \theta)$ is positive definite. The derivative of $V$ along the solution of the equation of motion, denoted by $\dot{V}$, is

$$
\dot{V}=\dot{\theta}^{T} B u_{1}
$$

where we have used the fact that $C(\theta, \dot{\theta})$ can be chosen (only $C(\theta, \dot{\theta}) \dot{\theta}$ is unique, but $C(\theta, \dot{\theta})$ is not) so that $\frac{1}{2} \dot{M}-C(\theta, \dot{\theta})$ is skew symmetric (a fact that was used in $[2,22,23,24,25]$ and many others). Integrating both sides of (29) and using the fact that $V$ is positive definite. it follows that the map from $u_{1}$ to $B^{T} \dot{\theta}=\dot{\theta}_{m}$ is passive. 


\subsubsection{Stabilization}

Once the passivity from $u_{1}$ to $B^{T} \dot{\theta}$ is established, a large family of feedback control law can be used to achieve I/O stability:

$$
u_{1}=u_{2}-\mathcal{C}_{v}\left(B^{T} \dot{\theta}\right)
$$

where $\mathcal{C}_{v}$ is any strictly passive system. Since the closed-loop system is the feedback connection of a passive system and a strictly passive system, by the Passivity Theorem, the map from $u_{2}$ to $B^{T} \dot{\theta}$ is $L_{2}$-stable. Furthermore, if $u_{2}=0$, we can conclude from (22) and the Invariance Principle that $(\theta, \dot{\theta})$ converges to the largest invariant set in $\left\{(\theta, \dot{\theta}): B^{T} \dot{\theta}=0\right\}$. To see this, recall that the strict passivity of $\mathcal{C}_{v}$ means

$$
\int_{0}^{T} w^{T} \mathcal{C}_{v}(w) d t \geq-\gamma^{2}+\eta \int_{0}^{T}\|w\|^{2} d t
$$

for any $w \in L_{2_{e}}$. Substituting $B^{T} \dot{\theta}$ into $w$, and noting that the left hand side of (24) asymptotically vanishes due to (22), it follows that $B^{T} \dot{\theta} \in L_{2}$. Now by applying the standard argument that $\vec{\theta}$ are uniformly bounded, we can conclude $B^{T} \dot{\theta} \rightarrow 0$ asymptotically. Furthermore, since all higher derivatives of $\dot{\theta}$ are uniformly bounded, all higher derivatives of $B^{T} \dot{\theta}$ also tend to zero asymptotically. If the closed loop system is zero state detectable from $B^{T} \dot{\theta}$, then the zero error state is asymptotically stable. If the detectability is global, then so

$\because$ is the asymptotic stability.

Under the following assumptions (slight generalization of the conditions in [4] and including the approximate model in [18] as a special case), the zero state detectability can be shown:

1. The mass matrix $M$ is of the special form

$$
M(\theta)=\left[\begin{array}{cc}
M_{11}\left(\theta_{\ell}\right) & M_{12}\left(\theta_{\ell}\right) \\
M_{12}{ }^{T}\left(\theta_{\ell}\right) & M_{22}
\end{array}\right] .
$$

This assumption is valid when the motor is symmetric about its axis of rotation; otherwise, all four blocks would depend on both $\theta_{\ell}$ and $\theta_{m}$ [26].

2. The gravity load $g$ and elastic coupling $k$ are given by (11) and (12).

3. $k$ is diagonal (i.e., $k_{i}$ only depends on $\theta_{\ell i}$ and $\theta_{m i}$ ).

4. $\nabla_{\theta_{\ell}} k\left(\theta_{\ell}, \theta_{m}\right)$ is positive semi-definite for all $\theta_{\ell}$ and $\theta_{m}$, and $\left(\theta_{\ell}, \theta_{m}\right)$ for which $\nabla_{\theta_{\ell}} k\left(\theta_{\ell}, \theta_{m}\right)$ loses rank are discrete.

To see how this set of assumptions lead to detectability, substitute $\ddot{\theta}_{m}=0$ into the dynamical equation (1), then we have

$$
\begin{aligned}
M_{11}\left(\theta_{\ell}\right) \ddot{\theta}_{\ell} & =-C_{1}\left(\theta_{\ell}, \dot{\theta}_{\ell}\right) \dot{\theta}_{\ell}-g_{1}\left(\theta_{\ell}\right)+g_{1}\left(\theta_{\ell_{\text {des }}}\right)-N k_{1}\left(\theta_{\ell}, \theta_{m}\right)+N k_{1}\left(\theta_{\ell_{\text {des }}}, \theta_{m_{\text {des }}}\right) \\
M_{12}{ }^{T}\left(\theta_{\ell}\right) \ddot{\theta}_{\ell} & =k_{1}\left(\theta_{\ell}, \theta_{m}\right)-k_{1}\left(\theta_{\ell_{\text {des }}}, \theta_{m_{\text {des }}}\right)-K_{p} \Delta \theta_{m} .
\end{aligned}
$$


Differentiate (26) once more, we have

$$
\sum_{i=1}^{n} \frac{\partial M_{12}{ }^{T}\left(\theta_{\ell}\right)}{\partial \theta_{\ell_{i}}} \theta_{\ell_{i}} \ddot{\theta}_{\ell}+M_{12}{ }^{T}\left(\theta_{\ell}\right) \dddot{\theta}_{\ell}=\nabla_{\theta_{\ell}} k_{1}\left(\theta_{\ell}, \theta_{m}\right) \dot{\theta}_{\ell}
$$

It has been independently pointed out in [26] and [4] (the former is for the exact case) that $M_{12}$ is strictly upper triangular. Applying this fact and assumptions 3 and 4 to (27), it follows that $\dot{\theta}_{\ell}=0$. Substituting back into (15), we obtain

$$
g(\theta)-g\left(\theta_{\text {des }}\right)+k(\theta)-k\left(\theta_{\text {des }}\right)+B K_{p} B^{T} \Delta \theta=0 .
$$

From the assumption that $K_{p}$ has been chosen sufficiently large as in (17), (28) implies local asymptotic stability. If (17) holds uniformly for all $\theta_{\text {des }}$, then the asymptotic stability is in fact global.

For the general model, the observability condition can be checked for the linearized system. First set $u_{2}(t)=0$ and $B^{T} \dot{\theta}(t)=B^{T} \ddot{\theta}(t)=0$. From linearized closed-loop equation of motion, we have

$$
B^{T} M\left(\theta_{\text {des }}\right)^{-1}\left(\nabla_{\theta} g\left(\theta_{\text {des }}\right)+\nabla_{\theta} k\left(\theta_{\text {des }}\right)+B K_{p} B^{T}\right) \Delta \theta=B^{T} M\left(\theta_{\text {des }}\right)^{-1} \hat{K} \Delta \theta=0 .
$$

Differentiating this equation twice more and use the equation of motion again, we have

$$
\begin{aligned}
B^{T} M\left(\theta_{\text {des }}\right)^{-1} \hat{K} \dot{\theta} & =0 \\
B^{T} M\left(\theta_{\text {des }}\right)^{-1} \hat{K} M\left(\theta_{\text {des }}\right)^{-1} \hat{K} \Delta \theta & =0 .
\end{aligned}
$$

These equations together imply the full state is identically zero if and only if $M\left(\theta_{\text {des }}\right)-\hat{K}$ is nonsingular, where $\hat{K} \triangleq \nabla_{\theta} g\left(\theta_{\text {des }}\right)+\nabla_{\theta} k\left(\theta_{\text {des }}\right)+B K_{p} B^{T}$.

From the above analysis, it is clear that under fairly mild conditions, the zero error state of the closed-loop system is globally asymptotic stable. But which $\mathcal{C}_{v}$ should one choose among the many possibilities in order to enhance a specified performance measure? This appears to be a hard question in general. We shall again encounter the same question in the next section. At the present, we do have some intuitive rules of thumb for the selection of $\mathcal{C}_{v}$. The simplest choice of $\mathcal{C}_{v}$ would be just a constant gain. Then the closed-loop control law is of the PD type (but only the motor variables are fed back). As demonstrated in simulation in [27], in contrast to the fully actuated robots, large PD gains degrade the closed-loop performance in terms of the settling time and amplitude of oscillation. This is due to the fact that the zeros in the $u_{1}$ to $\dot{\theta}_{m}$ system are on the $j \omega$-axis, high gains would then drive some of the poles toward these zeros and the response would become increasingly oscillatory. It is intuitively plausible to choose $\mathcal{C}_{v}$ to be an SPR (i.e., linear time invariant and strictly passive) compensator where the gain is concentrated at the open-loop resonant frequencies (so that a small oscillation in $\dot{\theta}_{m}$ will cause a large corrective action) and at the disturbance frequencies (as in notch filters). In simulation [27], much improvement is obtained by using this approach. This idea is similar to a common practice in servo control where a band pass or high pass filter is used in the motor velocity loop (usually analog), in addition to the usual PID loop, to improve performance in the higher frequency range (for example, see the servo controller for space shuttle remote manipulator system in [28]). For the type of 
systems considered here, we can be more specific about the class of filters that can be tuned for increased performance without affecting the stability.

In the feedforward, the only model-dependent information that is required is the gravity load and spring coupling. If this information is inexact, then $u_{2}$ in (23) is a nonzero constant. Since local internal asymptotic stability implies bounded-input/bounded-output (BIBO) stability for sufficiently small initial error, the output error $\theta_{\ell}-\theta_{\ell_{\text {de }}}$ is also proportionally bounded, and the internal states would remain bounded. In Section 8 , we will adaptively update this constant; not surprisingly, the resulting control law is of the standard proportional-integral-derivative (PID) structure.

In the case that the full state is available, an interesting question arises: How can $\theta_{\ell}$ and $\dot{\theta}_{\ell}$ be included in this passive control framework? A reasonable approach would be to find another output which is independent from $\dot{\theta}_{m}$ and passive with respect to $u_{2}$ (i.e., after the $\Delta \theta_{m}$ and $\dot{\theta}_{m}$ loops have been closed as described above). Then an additional strictly passive feedback can be applied to enhance transient performance. Finding an additional passive output for a linear system of the form $\dot{x}=A x+B u$ is straightforward: solve the Lyapunov Equation $A^{T} P+P A+Q$ for some $Q>0$, then choose the output map to be $C=B^{T} P$. A general procedure for nonlinear systems such as the flexible joint robots is unknown at the present.

\subsubsection{A Simplified Dynamical Model}

The exact model for flexibly jointed robots is not exact linearizable [29]. In [18], a simplified model for flexibly jointed robots was proposed. This model ignores the gyroscopic forces due the motion of rotating motors in the inertial space. Based on this model, an exact linearizing control law was obtained. The simplified and full models have been compared in [17] based on the parameters of a PUMA 560 robot and it is concluded that the approximate model is a very good one for earth bound applications (when the arm is mounted on a mobile base, the effect is far more drastic). The space shuttle remote manipulator system is also modeled under this assumption [28]. In this subsection, we consider the stability analysis and control design discussed above as applied to this simplified model.

The simplified model is of the form

$$
\begin{aligned}
M_{1}\left(\theta_{\ell}\right) \ddot{\theta}_{\ell}+C_{1}\left(\theta_{\ell}, \dot{\theta}_{\ell}\right) \dot{\theta}_{\ell}+g_{1}\left(\theta_{\ell}\right)+N k_{1}\left(N \theta_{\ell}-\theta_{m}\right) & =0 \\
I_{m} \ddot{\theta}_{m}-k_{1}\left(N \theta_{\ell}-\theta_{m}\right) & =u .
\end{aligned}
$$

Given the desired link angle vector $\theta_{\ell_{\text {des }}}$, the steps in section 4.1 can be followed to obtain the feedforward control $u_{f f}$ and desired motor angle vector $\theta_{m_{d e s}}$ for the error system:

$$
\begin{aligned}
u_{f f} & =\frac{g_{1}\left(\theta_{\ell_{\text {des }}}\right)}{N} \\
\theta_{m_{\text {des }}} & =N \theta_{\ell_{\text {des }}}-k_{1}^{-1}\left(-\frac{g_{1}\left(\theta_{\ell_{\text {des }}}\right)}{N}\right)
\end{aligned}
$$

where $k_{1}$ is assumed to be globally invertible. The spring model for $k_{1}$ is usually assumed to be diagonal (i.e., the $i$ th component of $k_{1}(x)$ only depends on $x_{i}$ ) and each component is monotonically increasing. Hence, the invertibility assumption on $k_{1}$ is a very reasonable one. 
The error system is described by

$$
\begin{aligned}
& M_{1}\left(\theta_{\ell}\right) \ddot{\theta}_{\ell}+C_{1}\left(\theta_{\ell}, \dot{\theta}_{\ell}\right) \dot{\theta}_{\ell}+g_{1}\left(\theta_{\ell}\right)-g_{1}\left(\theta_{\ell_{\text {des }}}\right) \\
& \quad+N k_{1}\left(N \theta_{\ell}-\theta_{m}\right)-N k_{1}\left(N \theta_{\ell_{\text {des }}}-\theta_{m_{\text {des }}}\right)=0 \\
& I_{m} \ddot{\theta}_{m}-k_{1}\left(N \theta_{\ell}-\theta_{m}\right)+k_{1}\left(N \theta_{\ell_{\text {des }}}-\theta_{m_{\text {des }}}\right)=u_{0}
\end{aligned}
$$

where $u=u_{0}+u_{f f}$ has been used. As in section 4.1.1, in order to show passivity, we introduce a proportional feedback to create a positive definite potential energy at the desired set point:

$$
u_{o}=u_{1}-K_{p} \Delta \theta_{m}
$$

Now, assume

$$
\left[\begin{array}{cc}
\nabla_{\theta} g_{1}\left(\theta_{\ell_{\text {des }}}\right)+N^{2} \nabla_{\theta} k_{1}\left(N \theta_{\ell_{\text {des }}}-\theta_{m_{\text {des }}}\right) & -N \nabla_{\theta} k_{1}\left(N \theta_{\ell_{\text {des }}}-\theta_{m_{\text {des }}}\right) \\
-N \nabla_{\theta} k_{1}\left(N \theta_{\ell_{\text {des }}}-\theta_{m_{\text {des }}}\right) & K_{p}+\nabla_{\theta} k_{1}\left(N \theta_{\ell_{\text {des }}}-\theta_{m_{\text {des }}}\right)
\end{array}\right]>0 .
$$

This condition is satisfied if the spring is sufficiently stiff compared to the gravity load (typically a reasonable assumption especially for geared robots) and $K_{p}$ is sufficiently large in the following sense:

$$
\begin{gathered}
N^{2} \nabla_{\theta} k_{1}\left(N \theta_{\ell_{\text {des }}}-\theta_{m_{\text {des }}}\right)>-\nabla_{\theta} g_{1}\left(\theta_{\ell_{\text {des }}}\right) \\
\sigma_{\min }\left(K_{p}\right)>\frac{N^{2}\left\|\nabla_{\theta} k_{1}\left(N \theta_{\ell_{\text {des }}}-\theta_{m_{\text {des }}}\right)\right\|^{2}}{\sigma_{\min }\left(N^{2} \nabla_{\theta} k_{1}\left(N \theta_{\ell_{\text {des }}}-\theta_{m_{\text {des }}}\right)+\nabla_{\theta} g_{1}\left(\theta_{\ell_{\text {des }}}\right)\right)}-\sigma_{\min }\left(\nabla_{\theta} k_{1}\left(N \theta_{\ell_{\text {des }}}-\theta_{m_{\text {des }}}\right)\right) .
\end{gathered}
$$

With the storage function

$$
V=\frac{1}{2} \dot{\theta}_{\ell}{ }^{T} M_{1}\left(\theta_{\ell}\right) \dot{\theta}_{\ell}+\frac{1}{2} \dot{\theta}_{m}{ }^{T} I_{m} \dot{\theta}_{m}+U\left(\Delta \theta_{\ell}, \Delta \theta_{m}\right)
$$

where

$$
\begin{aligned}
U\left(\Delta \theta_{\ell}, \Delta \theta_{m}\right)=G_{1}\left(\Delta \theta_{\ell}+\theta_{\ell_{\text {des }}}\right)-G_{1}\left(\theta_{\ell_{\text {des }}}\right)-g_{1}\left(\theta_{\ell_{\text {des }}}\right)^{T} \Delta \theta_{\ell} \\
+ \\
+K_{1}\left(N \Delta \theta_{\ell}-\Delta \theta_{m}+N \theta_{\ell_{\text {des }}}-\theta_{m_{\text {des }}}\right)-K_{1}\left(N \theta_{\ell_{\text {des }}}-\theta_{m_{\text {des }}}\right) \\
\\
-k_{1}\left(N \theta_{\ell_{\text {des }}}-\theta_{m_{\text {des }}}\right)^{T}\left(N \Delta \theta_{\ell}-\Delta \theta_{m}\right)+\frac{1}{2} \Delta \theta_{m}{ }^{T} K_{p} \Delta \theta_{m} .
\end{aligned}
$$

The scalar functions $K_{1}$ and $G_{1}$ are the spring potential energy and gravity potential energy, respectively. Again use the skew symmetric property of $\frac{1}{2} \dot{M}_{1}-C_{1}$; it follows that the derivative of $V$ along the solution trajectory of $(29)-(30)$ is

$$
\dot{V}=\dot{\theta}_{m}{ }^{T} u_{1}
$$

which implies that the map from $u_{1}$ to $\dot{\theta}_{m}$ is passive.

The final step is to choose a motor velocity feedback for stabilization. Again by the Passivity Theorem, $u_{1}$ can be chosen as

$$
u_{1}=u_{2}-\mathcal{C}_{v}\left(\dot{\theta}_{m}\right)
$$

where $\mathcal{C}_{v}$ is strictly passive, the closed-loop system is $L_{2}$ I/O stable from $u_{2}$ to $\dot{\theta}_{m}$.

Since the simplified model in this section satisfies all the assumptions stated in the last section, global asymptotic stability of the zero error equilibrium follows from the I/O stability. 


\section{Application to a Single Flexible Link}

Consider the linearized model for a single link flexible link $[30,31]$ discretized in terms of the natural modes:

$$
\ddot{q}+\Omega^{2} q=b u
$$

where $q$ is the modal amplitude, $u$ is the hub torque, and

$$
\Omega^{2}=\left[\begin{array}{cc}
0_{1 \times 1} & 0_{1 \times n} \\
0_{n \times 1} & \operatorname{diag}_{n \times n}\left\{\omega_{i}^{2}\right\}
\end{array}\right], \quad b^{T}=\frac{1}{\rho}\left[\psi_{0}^{\prime}(0) \quad \psi_{1}^{\prime}(0) \quad \ldots \quad \psi_{n}^{\prime}(0)\right]
$$

where $\rho$ is the link density (over unit length), $\omega_{i}$ 's are the natural modal frequencies and $\psi_{i}$ 's are the corresponding mode shapes. Spatial derivatives are denoted by '. Here we consider only an $(n+1)$-mode approximation to avoid the technicality associated with infinite dimensional systems. For a discussion in the infinite dimensional context, see [31]. Note also that the nonlinear model in [31] is of the same form as (6) in the flexible joint robot case. The same analysis as in the previous section can be applied. Here we will concentrate on the linearized model.

Let $x=[q, \dot{q}]^{T}$. The state space equation is

$$
\dot{x}=\left[\begin{array}{cc}
0 & I \\
-\Omega^{2} & 0
\end{array}\right] x+\left[\begin{array}{l}
0 \\
b
\end{array}\right] u .
$$

Assume that the hub angle and angular velocity can be measured. The corresponding output equations are

$$
\begin{aligned}
& y_{p}=\left[\begin{array}{ll}
b^{T} & 0
\end{array}\right] x \\
& y_{v}=\left[\begin{array}{ll}
0 & b^{T}
\end{array}\right] x
\end{aligned}
$$

where $y_{p}$ and $y_{v}$ are proportional to the hub angular position and velocity, respectively.

\subsection{Feedforward Compensation based on Steady State Analysis}

Suppose the output of interest is the scalar variable

$$
y=C q
$$

Consider the set point control problem of steering an arbitrary initial state $(q(0), \dot{q}(0))$ to a steady state which corresponds to a specified desired output $y_{\text {des. }}$. As in (3)-(4), we are interested in finding a full state set point $q_{d e s}$ which maps to the desired output $y_{\text {des }}$, and a feedforward $u_{f f}$ that cancels the extra terms in the error dynamical equation for $\Delta q=q-q_{d e s}$. This means $q_{d e s}$ and $u_{f f}$ must satisfy the following equations:

$$
\begin{aligned}
\Omega^{2} q_{d e s}-b u f f & =0 \\
C q_{d e s} & =y_{\text {des }} .
\end{aligned}
$$


Assume the leading components in $b$ and $C, b_{0}$ and $c_{0}$, respectively, are nonzero. Then the model matching equations (44)-(45) imply

$$
\begin{aligned}
u_{f f} & =0 \\
q_{\text {des } 0} & =\frac{y_{\text {des }}}{c_{0}} \\
q_{\text {des } i} & =0 \quad \text { for } i \geq 1 .
\end{aligned}
$$

The error equation is then governed by

$$
\begin{aligned}
\ddot{q}+\Omega^{2} \Delta q & =b u \\
\Delta y_{p} & =b^{T} \Delta q
\end{aligned}
$$

where $\Delta q=q-q_{d e s}$.

\subsection{Passivity}

In the error dynamical equation, $\Omega^{2}$ is only positive semidefinite. For internal stability, cf. section 5.3 below, it is important that the stiffness matrix is positive definite. To achieve this, a proportional feedback loop is first closed:

$$
u=u_{1}-k_{p} b^{T} \Delta q
$$

The effective closed-loop stiffness matrix is then

$$
\tilde{\Omega}^{2}=\Omega^{2}+k_{p} b b^{T}
$$

Since it is assumed that $b_{0} \neq 0, \widetilde{\Omega}^{2}$ is positive definite for any $k_{p}>0$.

To show the mapping $u_{1}$ to $b^{T} \dot{q}$ is passive, consider the storage function

$$
V(x)=\frac{1}{2}\|\dot{q}\|^{2}+\frac{1}{2} q^{T} \tilde{\Omega}^{2} q
$$

It is easily verified that the derivative of $V$ along the solution is $\dot{V}=\left(b^{T} \dot{q}\right)^{T} u_{1}$. The passivity from $u_{1}$ to $b^{T} \dot{q}$ follows from the fact that $V$ is a positive function.

\subsection{Stabilization}

For the open-loop error system (49), the controllability matrix, after reordering the columns, is

$$
\mathcal{C}=\left[\begin{array}{cc}
0 & \mathcal{C}_{0} \\
\mathcal{C}_{0} & 0
\end{array}\right]
$$

with

$$
C_{0}=\left[\begin{array}{lllll}
b & -\Omega^{2} b & \Omega^{4} b & \ldots & \left(-\Omega^{2}\right)^{n} b
\end{array}\right] .
$$

Assume the modal frequencies are all distinct and every component of $b$ is nonzero, then $\mathcal{C}_{0}$ is invertible which means that the system is controllable. 
The observability matrix with respect to $y_{v}$ for the open-loop error system is

$$
\mathcal{O}=\left[\begin{array}{cc}
0 & -\Omega^{2} \mathcal{C}_{0} \\
\mathcal{C}_{0} & 0
\end{array}\right]
$$

Since $\Omega^{2}$ is singular, $\mathcal{O}$ is singular which means that the system is not observable from $y_{v}$. However, with the proportional feedback of the motor position as in $(51), \Omega^{2}$ in the observability matrix is replaced

$$
\tilde{\Omega}^{2}=\Omega^{2}+k_{p} b b^{T}
$$

Since $\tilde{\Omega}^{2}$ is nonsingular, the observability matrix is also nonsingular and the system is observable.

By the Passivity Theorem, the hub velocity loop can be closed with any strictly passive feedback $\mathcal{C}_{v}$, i.e.,

$$
u_{1}=u_{2}-\mathcal{C}_{v}\left(b^{T} \dot{q}\right),
$$

and the resulting closed-loop system is $L_{2}$-stable from $u_{2}$ to $b^{T} \dot{q}$. For the internal asymptotic stability, we need stabilizability and detectability. From the analysis above, it is evident that if the poles of $\mathcal{C}_{v}$ do not cancel with the zeros of the system with proportional feedback, then the overall closed-loop system is controllable and observable, and, therefore, internally asymptotically stable.

It is tempting to choose $\mathcal{C}_{v}$ to be an SPR filter which, over certain bandwidth, approximates the plant inverse (this is possible since the plant is passive, therefore, minimum phase). Then the I/O map from $u_{2}$ to $b^{T} \dot{q}$ is approximately constant in that frequency range. This would result in an excellent $I / O$ response; however, the internal state becomes almost unobservable which means a very poor internal state response. This has indeed been observed experimentally, where excellent step response is obtained at the hub but the beam oscillates at a frequency corresponding to the pair of zeros with the lowest frequency.

\section{Application to Fully Actuated Robots}

The passivity property of fully actuated robots has been much exploited in recent years, starting from the path breaking work in [2] to many later extensions in, for example, [22, 23, $24,32]$ and many others. This section briefly reviews some of these results and shows how they fit into the framework outlined in Section 3.

The equation of motion for a fully actuated arm is the same as that for the flexible joint robot (5) except for $B=I$ and $k=0$ :

$$
M(\theta) \ddot{\theta}+C(\theta, \dot{\theta}) \dot{\theta}+g(\theta)=u .
$$

Consider the set point control problem, i.e., the control objective is to steer an arbitrary initial condition $(\theta(0), \dot{\theta}(0))$ to a specified set point $\left(\theta_{\text {des }}, 0\right)$.

The first step is to choose a feedforward control to form the error system

$$
u=u_{0}+u_{f f}
$$


where $u_{f f}$ may either be the gravity load cancellation or the gravity load at the desired set point:

$$
\begin{aligned}
& u_{f f}=g\left(\theta_{\text {des }}\right) \quad \text { or } \\
& u_{f f}=g(\theta) .
\end{aligned}
$$

In both cases, a position feedback loop needs to be closed to ensure a positive definite stiffness:

$$
u_{o}=u_{1}-K_{p} \Delta \theta
$$

where $K_{p}$ is positive definite. In the first case, $K_{p}$ should be chosen large enough so that the combination with the gravity potential energy is positive definite. To show the passivity from $u_{1}$ to $\dot{\theta}$, the following storage function can be used:

$$
V(\theta, \dot{\theta})=\frac{1}{2} \dot{\theta}^{T} M(\theta) \dot{\theta}+U(\Delta \theta)
$$

where $U$ is the total potential energy (including the position feedback loop)

$$
\begin{aligned}
& U(\Delta \theta)=\frac{1}{2} \Delta \theta^{T} K_{p} \Delta \theta+G\left(\Delta \theta+\theta_{\text {des }}\right)-G\left(\theta_{\text {des }}\right)-g\left(\theta_{\text {des }}\right)^{T} \Delta \theta \quad \text { for (53) } \\
& U(\Delta \theta)=\frac{1}{2} \Delta \theta^{T} K_{p} \Delta \theta . \quad \text { for (54) }
\end{aligned}
$$

Now, any strictly passive feedback from $\dot{\theta}$ to $u_{1}$ can be used:

$$
u_{1}=u_{2}-\mathcal{C}_{v}(\dot{\theta}) \text {. }
$$

By the Passivity Theorem, the closed-loop is $L_{2}$-stable from $u_{2}$ to $\dot{\theta}$. Since the stiffness term is globally positive definite, $\dot{\theta}$ is globally zero state detectable. With only the position feedback loop, the system is globally controllable (since $u$ can always be chosen as an exact linearizing controller). If the velocity loop is exponentially stable, then the stabilizability is preserved, hence the zero equilibrium of the error system is globally asymptotically stable.

\section{Tracking Control Problem}

So far we have considered only the set point control problem. Another important aspect of the control design is the output trajectory tracking problem. An intuitive approach is to simply replace $\dot{\theta}$ by $\Delta \dot{\theta}$ in the set point controller with the hope that a well tuned set point controller would also imply good tracking. In this section, we will both justify and modify this intuitive approach.

Given the general dynamical equation (1), consider the problem of finding a feedback control $u$ so that the output $y=C \theta$ tracks an arbitrary trajectory $y_{d e s}$ asymptotically. A natural extension of the set point control approach presented before is to express the system dynamics in the error coordinate and choose a feedforward control $u_{f f}$ to cancel the extra terms in the dynamics, assuming that this is possible:

$$
M(\theta) \Delta \ddot{\theta}+C(\theta, \dot{\theta}) \Delta \dot{\theta}+f(\theta)-f\left(\theta_{\text {des }}\right)=B u_{0}
$$


where $u=u_{o}+u_{f f}$ has been used and

$$
B u_{f f}=M(\theta) \ddot{\theta}_{\text {des }}+C(\theta, \dot{\theta}) \dot{\theta}_{\text {des }}+f\left(\theta_{\text {des }}\right)
$$

is assumed to have a solution, given $y_{\text {des }}(t)=C \theta_{\text {des }}(t), t \geq 0$ (this issue is discussed in greater detail in Section 7.0.4). Note that in contrast to the set point control case, not only is the model information required in the feedforward but, in general, the full state measurements as well.

An important extension of $(57)$ is to add to $\dot{\theta}_{\text {des }}$ with an error feedback, $\ell(\Delta \theta, \Delta \dot{\theta})$ (assume the equation is solvable). Then the feedforward to be solved is

$$
B u_{f f}=M(\theta)\left(\ddot{\theta}_{d e s}-\ell(\Delta \theta, \Delta \dot{\theta})\right)+C(\theta, \dot{\theta}) \dot{\theta}_{d e s}+f\left(\theta_{d e s}\right)
$$

The solvability of this equation in the flexible joint robot case is discussed in section 7.0.4. The error equation with this feedforward becomes

$$
M(\theta) \Delta \ddot{\theta}+M(\theta) \ell(\Delta \theta, \Delta \dot{\theta})+C(\theta, \dot{\theta}) \Delta \dot{\theta}+f(\theta)-f\left(\theta_{d e s}\right)=B u_{o}
$$

The additional term $\ell$ can now be chosen to augment performance (this is especially effective if $M$ strongly couples different degrees of freedom).

Next close a position loop:

$$
u_{o}=u_{1}-K_{p} B^{T} \Delta \theta
$$

where it is assumed that $K_{p}$ can be chosen sufficiently large so that $B K_{p} B^{T}+\nabla_{\theta} f\left(\theta_{\text {des }}\right)>0$ (same as the set point case). The problem is that $f\left(\theta_{\text {des }}\right)$ is now time varying and, consequently, the passivity property from $u_{1}$ to $\Delta \dot{\theta}$ cannot be easily shown as before (an exception is when $f$ is linear, a fact we shall use in section 7.0.2). There are three approaches to this issue:

1. The only time varying term in the error system is due to $\theta_{\text {des }}$. For each fixed time, the same passivity analysis as before can be applied to show local asymptotic stability. By applying a well known theorem for time varying systems [33], closed loop asymptotic stability is preserved if $\theta_{\text {des }}$ is sufficiently slow time varying.

2. If the feedforward torque, $u_{f f}$, is chosen to compensate for $g(\theta)$ rather than for $g\left(\theta_{\text {des }}\right)$, provided that it is solvable, then the passivity analysis can again be applied.

3. Define a new output $z=B^{T} \dot{\theta}+\mu B^{T} \theta$ where $\mu$ is a small positive parameter. If $B=I$ (full actuation case) or there is inherent structural damping $D$ such that $D+B K_{v} B^{T}$ is positive definite for some $K_{v}>0$, then the map from $u_{1}$ to $z$ is passive for $\mu$ sufficiently small, and the same passivity analysis can be applied.

In the remainder of this section, we will elaborate on each of these approaches, and also discuss in detail the solution of the feedforward torque. 


\subsubsection{Tracking for Slowly Varying Trajectories}

To apply the stability result for slowly time varying systems, the feedforward in (57) needs to be slightly modified to

$$
B u_{f f}=M(\theta) \ddot{\theta}_{d e s}+C(\theta, \dot{\theta}) \dot{\theta}_{d e s}+C\left(\theta, \dot{\theta}_{\text {des }}\right) \Delta \dot{\theta}+f\left(\theta_{d e s}\right) .
$$

Then the error equation becomes

$$
M(\theta) \Delta \ddot{\theta}+C(\theta, \Delta \dot{\theta}) \Delta \dot{\theta}+f(\theta)-f\left(\theta_{\text {des }}\right)=B u_{o} .
$$

In $(61)$, the only time varying quantities are $\theta_{\text {des }}$ and $\dot{\theta}_{\text {des }}$. If they are "frozen" at a particular constant value $\left(\theta_{\text {des }}, \dot{\theta}_{\text {des }}\right)=\left(\theta_{\text {des }}(T), \dot{\theta}_{\text {des }}(T)\right)$ where $T \geq 0$ is a constant, then the derivative of the following scalar function

$V(\Delta \theta, \Delta \dot{\theta})=\frac{1}{2} \Delta \dot{\theta}^{T} M\left(\Delta \theta+\theta_{\text {des }}\right) \Delta \dot{\theta}+U\left(\Delta \theta+\theta_{\text {des }}\right)-\Delta \theta^{T} f\left(\theta_{\text {des }}\right)-U\left(\theta_{\text {des }}\right)+\frac{1}{2} \Delta \theta^{T} B K_{p} B^{T} \Delta \theta$

is $\dot{V}=\Delta \dot{\theta}^{T} B u_{1}$, where $U$ is the potential energy corresponding to $f$ and $B K_{p} B^{T}+\nabla_{\theta} f\left(\theta_{\text {des }}\right)$ is assumed to be positive definite uniformly in $\theta_{\text {des. }}$. Hence, the stabilizing control law design based on the passivity approach as described in the previous sections (with $\dot{\theta}$ replaced by $\Delta \dot{\theta})$ stabilizes all frozen systems. Under the additional assumption that the frozen systems are locally uniformly (with respect to $T$ ) exponentially stable, a Theorem on slowly time varying systems as stated in [33, Theorem 5.6.6] can be applied to show local exponential stability of the closed loop system provided $\sup _{t} \max \left\{\dot{\theta}_{\text {des }}(t), \vec{\theta}_{\text {des }}(t)\right\}$ is sufficiently small. Simulations in [27] confirm this result, where a slowly time varying sinusoid can be closely tracked, but not a fast time varying sinusoid.

\subsubsection{Tracking by Direct Compensation}

Another possibility is to directly compensate for part of $f(\theta)$ in (56). The feedforward torque that needs to be solved is now

$$
B u_{f f}=M(\theta) \bar{\theta}_{\text {des }}+C(\theta, \dot{\theta}) \dot{\theta}_{\text {des }}+f_{1}(\theta)+F \theta_{\text {des }}
$$

where we have decomposed $f(\theta)$ according to $f(\theta)=f_{1}(\theta)+F \theta$ where $F$ is a square matrix. The reason that we decompose $f$ in this fashion is related to the solvability of $(62)$ (see section 7.0.4 for detail).

Assume that a solution exists, then the error equation is of the form

$$
M(\theta) \Delta \ddot{\theta}+C(\theta, \dot{\theta}) \Delta \dot{\theta}+F \Delta \theta=B u_{o} .
$$

Now the same passivity analysis as before can be applied for the control law

$$
u_{o}=-K_{p} B^{T} \Delta \theta-\mathcal{C}_{v}(\Delta \dot{\theta})
$$

for any strictly passive $\mathcal{C}_{v}$. 


\subsubsection{Tracking by Output Modification}

Even in the local version of (56), with $f(\theta)-f\left(\theta_{\text {des }}\right)$ replaced by $\nabla_{\theta} f\left(\theta_{\text {des }}\right) \Delta \theta, \nabla_{\theta} f\left(\theta_{\text {des }}\right)+$ $B K_{p} B^{T}>0$ for some $K_{p}$, the map from $u_{o}$ to $B^{T} \Delta \theta$ is still not passive in general. This can be seen by evaluating the $L_{2}$ innerproduct between this input/output pair:

$$
\begin{aligned}
\int_{0}^{T}\left(B^{T} \Delta \dot{\theta}\right)^{T} u_{0} d t= & \int_{0}^{T} \Delta \dot{\theta}^{T}\left(M(\theta) \Delta \bar{\theta}+C(\theta, \dot{\theta}) \Delta \dot{\theta}+\nabla_{\theta} f\left(\theta_{\text {des }}\right) \Delta \theta\right) d t \\
= & \left.\frac{1}{2} \Delta \dot{\theta}^{T} M(\theta) \Delta \dot{\theta}\right|_{0} ^{T}+\left.\frac{1}{2} \Delta \theta^{T} \nabla_{\theta} f\left(\theta_{d e s}\right) \Delta \theta\right|_{0} ^{T} \\
& -\frac{1}{2} \int_{0}^{T} \Delta \theta^{T} \frac{d}{d t}\left(\nabla_{\theta} f\left(\theta_{\text {des }}\right)\right) \Delta \theta d t .
\end{aligned}
$$

Since $\frac{d}{d t}\left(\nabla_{\theta} f\left(\theta_{\text {des }}\right)\right)$ may be sign indefinite, the integral cannot be bounded below by a constant. To counter the effect of this last term, we consider adding a proportional feedback, $B^{T} \Delta \theta$. The contribution to the input/output innerproduct due to this addition is

$$
\begin{aligned}
\int_{0}^{T}\left(B^{T} \Delta \theta\right)^{T} u_{o} d t= & \int_{0}^{T} \Delta \theta^{T}\left(M(\theta) \Delta \vec{\theta}+C(\theta, \dot{\theta}) \Delta \dot{\theta}+\nabla_{\theta} f\left(\theta_{d e s}\right) \Delta \theta\right) d t \\
= & \left.\Delta \dot{\theta}^{T} M(\theta) \Delta \theta\right|_{0} ^{T}-\int_{0}^{T}\left(\Delta \dot{\theta}^{T} M(\theta) \Delta \dot{\theta}+\Delta \theta^{T}(\dot{M}(\theta, \dot{\theta})-C(\theta, \dot{\theta})) \Delta \dot{\theta}\right) d t \\
& \quad+\int_{0}^{T} \Delta \theta^{T} \nabla_{\theta} f\left(\theta_{\text {des }}\right) \Delta \theta d t .
\end{aligned}
$$

For the local analysis, we shall ignore the higher order term $(\dot{M}(\theta, \dot{\theta})-C(\theta, \dot{\theta}))$. Now, consider adding a static PD loop:

$$
u_{o}=-K_{p} B^{T} \Delta \theta-K_{v} B^{T} \Delta \dot{\theta}+u_{1} .
$$

Then the innerproduct between $u_{1}$ and $B^{T} \Delta \dot{\theta}$ is the same as (65) except $\nabla_{\theta} f\left(\theta_{d e s}\right)$ is replaced by $\nabla_{\theta} f\left(\theta_{\text {des }}\right)+B K_{p} B^{T}$ and there is an additional term:

$$
\int_{0}^{T} \Delta \dot{\theta}^{T} B K_{v} B^{T} \Delta \dot{\theta} d t
$$

The innerproduct between $u_{1}$ and $B^{T} \Delta \theta$ are the same as (66) except $\nabla_{\theta} f\left(\theta_{\text {des }}\right)$ is replaced by $\nabla_{\theta} f\left(\theta_{\text {des }}\right)+B K_{p} B^{T}$ and there is an additional term:

$$
\left.\frac{1}{2} \Delta \theta^{T} B K_{v} B^{T} \Delta \theta\right|_{0} ^{T}
$$

Now form the augmented output

$$
z=B^{T} \Delta \dot{\theta}+c B^{T} \Delta \theta
$$

For $c$ sufficiently small and $\theta_{\text {des }}$ sufficiently slowly time varying, all terms in $\int_{0}^{T} z^{T}(t) u_{1}(t) d t$ can be bounded below by a constant except for the integral involving the quadratic term in $\Delta \dot{\theta}$ which is

$$
\int_{0}^{T} \Delta \dot{\theta}^{T}\left(-c M(\theta)+B K_{v} B^{T}\right) \Delta \dot{\theta} d t
$$


Terms involving the error states at time $T$ can be lumped together in a quadratic form:

$$
\left[\begin{array}{c}
\Delta \theta(T) \\
\Delta \dot{\theta}(T)
\end{array}\right] T\left[\begin{array}{cc}
\frac{c}{2}\left(\nabla_{\theta} f+B K_{p} B^{T}\right) & c M \\
c M & \frac{1}{2} M
\end{array}\right]\left[\begin{array}{c}
\Delta \theta(T) \\
\Delta \dot{\theta}(T)
\end{array}\right]
$$

Since $M$ and $\nabla_{\theta} f+B K_{p} B^{T}$ are both positive definite, for $c$ sufficiently small, this term is positive.

Only the indefinite term in (68) prevents the map from $u_{1}$ to $z$ from being passive. There are two situations in which this term is also bounded below by a constant:

1. The arm is fully actuated, i.e., $B=I$. This approach is the same as in [34].

2. There is an inherent damping, $D \dot{\theta}$, which gives rise to the term $D \Delta \dot{\theta}$ in the error equation (the feedforward $u_{f f}$ needs to be modified accordingly). If $D+B K_{v} B^{T}$ is positive definite, then for $c$ sufficiently small, the integral is bounded below by a constant.

If either of the above situation holds, then the map from $u_{1}$ to $z$ is passive and the same analysis can be carried as before to generate stabilizing control laws based on passive map from $z$ to $u_{1}$. In the example in [27], it has been shown that link damping in a flexibly jointed robot allows tracking of a fast trajectory that could not be tracked when the damping is absent.

\subsubsection{Derivation of the Feedforward Compensation}

Flexible Joint Robot Case

To form the tracking error dynamic equation, we need to solve for $u_{f f}$ in either (57) or (62). In this section, we will consider this problem for the special cases of a flexible jointed robot and a single flexible link.

We will consider only the simplified flexible joint model given in (29)-(30); the general case is considerably more complicated. Suppose $y=\theta_{\ell}$. Then (57) involves solving for $\left(u_{f f}(t), \theta_{m_{\text {des }}}(t), \dot{\theta}_{m_{\text {des }}}(t)\right)$, given $\theta_{\ell_{\text {des }}}(t)$ and its higher time derivatives (as many as required) and $\left(\theta_{m}(t), \dot{\theta}_{m}(t), \theta_{\ell}(t), \dot{\theta}_{\ell}(t)\right)$, from the following set of equations:

$$
\begin{aligned}
M_{1}\left(\theta_{\ell}\right) \ddot{\theta}_{\ell_{\text {des }}}+C_{1}\left(\theta_{\ell}, \dot{\theta}_{\ell}\right) \dot{\theta}_{l_{\text {des }}}+g_{1}\left(\theta_{\ell_{\text {des }}}\right)+N k_{1}\left(N \theta_{\ell_{\text {des }}}-\theta_{m_{\text {des }}}\right) & =0 \\
I_{m} \ddot{\theta}_{m_{\text {des }}}-k_{1}\left(N \theta_{\ell_{\text {des }}}-\theta_{m_{\text {des }}}\right) & =u_{f f}
\end{aligned}
$$

Assuming $k_{1}$ is strictly monotonically increasing so an inverse function $k^{-1}$ exists. Assume $k_{1}$ is twice differentiable. Then $\theta_{m_{d e}}$ can be solved from (69):

$$
\theta_{m_{\text {des }}}=N \theta_{\ell_{\text {des }}}+k_{1}^{-1}\left(\frac{1}{N}\left[M_{1}\left(\theta_{\ell}\right) \ddot{\theta}_{\ell_{\text {des }}}+C_{1}\left(\theta_{\ell}, \dot{\theta}_{\ell}\right) \dot{\theta}_{\ell_{\text {des }}}+g_{1}\left(\theta_{\ell_{\text {des }}}\right)\right]\right) \text {. }
$$

To solve $u_{f f}$ from ( 70$), \vec{\theta}_{m_{\text {des }}}$ must first be computed. This can be done by differentiating (71) twice:

$$
\ddot{\theta}_{m_{d e s}}=N \ddot{\theta}_{\ell_{d e s}}+\frac{d^{2}}{d t^{2}}\left[k_{1}^{-1}\left(\frac{1}{N}\left[L_{1}\left(\theta_{i}\right) \ddot{\theta}_{l_{d e s}}+C_{1}\left(\theta_{\ell} \cdot \dot{\theta}_{\ell}\right) \dot{\theta}_{i_{d e s}}+g_{1}\left(\theta_{\ell_{d s}}\right)\right]\right)\right] .
$$


Note that the second term involves $\vec{\theta}_{\ell}$ and $\dddot{\theta}_{\ell}$, which can in turn be resolved using the dynamic equation (29) and its derivative. Finally, $u_{f f}$ can be computed from (70).

For the direct compensation case, cf. (62), the feedforward compensation equation based on the simplified flexible joint model is

$$
\begin{aligned}
& M_{1}\left(\theta_{\ell}\right) \ddot{\theta}_{l_{\text {des }}}+C_{1}\left(\theta_{\ell}, \dot{\theta}_{\ell}\right) \dot{\theta}_{\ell_{\text {des }}}+g_{1}\left(\theta_{\ell}\right) \\
& +N k_{2}\left(N \theta_{\ell}-\theta_{m}\right)+N K\left(N \theta_{\ell_{\text {des }}}-\theta_{m_{\text {des }}}\right)=0 \\
& I_{m} \ddot{\theta}_{m_{\text {des }}}-k_{2}\left(N \theta_{\ell}-\theta_{m}\right)-K\left(N \theta_{\ell_{\text {des }}}-\theta_{m_{\text {des }}}\right)=u_{f f}
\end{aligned}
$$

where $K$ is any square invertible matrix and $k_{2}$ is chosen from $k_{2}(x)=k_{1}(x)-K x$. Following similar steps as before, (73) can be used to solve for $\theta_{m_{d s e}}$ :

$$
\theta_{m_{\text {des }}}=\frac{K^{-1}}{N}\left(M_{1}\left(\theta_{\ell}\right) \ddot{\theta}_{\ell_{\text {des }}}+C_{1}\left(\theta_{\ell}, \dot{\theta}_{\ell}\right) \dot{\theta}_{\ell_{\text {des }}}+g_{1}\left(\theta_{\ell}\right)+N k_{2}\left(N \theta_{\ell}-\theta_{m}\right)\right)+N \theta_{\ell_{\text {des }}} .
$$

To solve for $u_{f f}$ from (74), again $\ddot{\theta}_{m_{d e} s}$ needs to be computed by directly twice differentiating

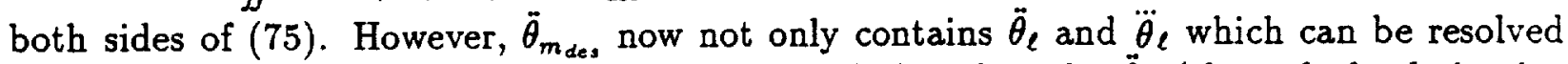
using the dynamical equation and its derivative as before, but also $\ddot{\theta}_{m}$ (through the derivative of $k_{2}$ ) which in turn depends on $u_{f f}$. Therefore, to solve $u_{f f}$, we need the invertibility of $\left(I+\left.K^{-1} \nabla_{x} k_{2}(x)\right|_{N \theta_{\ell}-\theta_{m}}\right)$ for all $\theta_{\ell}$ and $\theta_{m}$, which does not appear to be a severe limitation. Note that if the spring is assumed to be linear as common practiced in the literature, this additional assumption would not be needed.

For flexible joint robots, Eq. (58) can be solved in exactly the same fashion as above. A simple but useful choice of the function $\ell$ is simply

$$
\ell(\Delta \theta, \Delta \dot{\theta})=K_{p_{\ell}} \Delta \theta_{\ell}+K_{v_{\ell}} \Delta \dot{\theta}_{\ell}
$$

The closed loop equation is now of the following form

$$
\begin{aligned}
& M_{1}\left(\theta_{\ell}\right) \Delta \ddot{\theta}_{\ell}+C_{1}\left(\theta_{\ell}, \dot{\theta}_{\ell}\right) \Delta \dot{\theta}_{\ell}+g_{1}\left(\theta_{\ell}\right)-g_{1}\left(\theta_{\ell_{\text {des }}}\right) \\
& \quad+N k_{1}\left(N \theta_{\ell}-\theta_{m}\right)-N k_{1}\left(N \theta_{\ell_{\text {des }}}-\theta_{m_{\text {des }}}\right)+M_{1}\left(\theta_{\ell}\right)\left(K_{u_{\ell}} \Delta \dot{\theta}_{\ell}+K_{p_{\ell}} \Delta \theta_{\ell}\right)=0 \\
& I_{m} \Delta \ddot{\theta}_{m}-k_{1}\left(N \theta_{\ell}-\theta_{m}\right)+k_{1}\left(N \theta_{\ell_{\text {des }}}-\theta_{m_{\text {des }}}\right)+K_{p_{m}} \Delta \theta_{m}=u_{1}
\end{aligned}
$$

The system linearized about $(\Delta \theta, \Delta \dot{\theta})=(0,0)$ is passive between $u_{1}$ and $\Delta \dot{\theta}_{m}$ since the stiffness matrix

$$
\left[\begin{array}{cc}
\nabla g_{1}\left(\theta_{\ell_{\text {des }}}\right)+N^{2} \nabla k_{1}\left(N \theta_{\ell_{\text {des }}}-\theta_{m_{\text {des }}}\right)+M_{1}\left(\theta_{\ell_{\text {des }}}\right) K_{p_{\ell}} & -N \nabla k_{1}\left(N \theta_{\ell_{\text {des }}}-\theta_{m_{\text {dess }}}\right) \\
-N \nabla k_{1}\left(N \theta_{\ell_{\text {des }}}-\theta_{m_{\text {des }}}\right) & \nabla k_{1}\left(N \theta_{\ell_{\text {des }}}-\theta_{m_{\text {des }}}\right)+K_{p_{m}}
\end{array}\right]
$$

is positive definite. Hence, any strictly passive loop between $\Delta \dot{\theta}_{m}$ and $u_{1}$ can be closed to ensure closed loop asymptotic stability of the error system.

The purpose of the feedforward control can be thought of as winding up the spring torque so that the link dynamics is governed by

$$
\Delta \ddot{\theta}_{\ell}+K_{v_{\ell}} \Delta \dot{\theta}_{\ell}+K_{p_{\ell}} \Delta \theta_{\ell}=0 .
$$


The role of the feedback control is to produce the motor trajectory that is required for this feedforward.

Flexible Link Case

For the flexible link case, the situation is quite different since the acceleration of the actuated degrees of freedom are not decoupled from the acceleration of the unactuated degrees of freedom as in the simplified model of a flexibly jointed robot. We now need to solve for $\left(u_{f f}, q_{d e s}\right)$ given a desired output trajectory $y_{d e s}$ :

$$
\begin{aligned}
& \ddot{q}_{d e s}+\Omega^{2} q_{d e s}=B u_{f f} \\
& C q_{d e s}=y_{d e s}
\end{aligned}
$$

with the additional constraint that $u_{f f}$ needs to be uniformly bounded for implementability. This problem is almost identical to the inverse plant problem considered by [35], but here we solve for the desired plant trajectory rather than the actual plant trajectory. Consequently, while the control law obtained in [35] is entirely open-loop, here we have a feedback control structure.

To analyze the solution of (79)-(80), first express $q_{\text {des }}$ in the following form:

$$
q_{d e s}=C^{+} y_{d e s}+\tilde{C} \xi
$$

where $C^{+}=C^{T}\left(C C^{T}\right)^{-1}$ is the pseudo-inverse of $C$ and $\tilde{C}$ is the $n \times(n-m)$ full rank matrix that is annihilated by $C(C \tilde{C}=0)$. Note that $\tilde{C}$ can be formed by the linearly independent columns of $\left(I-C^{+} C\right)$, but $\tilde{C} \neq\left(I-C^{+} C\right)$ since $\tilde{C}$ is full rank.

Differentiating the equation twice, we have

$$
\ddot{q}_{d e s}=C^{+} \ddot{y}_{d e s}+\tilde{C} \tilde{\xi} .
$$

Substitute back into ( 79$)$ and assume $B$ is full rank, we can solve for $u_{f f}$.

$$
u_{f f}=\left(B^{T} B\right)^{-1}\left(B^{T} C^{+} \ddot{y}_{d e s}+B^{T} \tilde{C} \tilde{\xi}+B^{T} \Omega^{2}\left(C^{+} y_{d e s}+\tilde{C} \xi\right)\right) .
$$

To eliminate $u_{f f}$ from (79), we multiply $\tilde{B}$ (the annihilator of $B$ ) from the left; and using (81), we have

$$
\tilde{B} \tilde{C} \tilde{\xi}+\tilde{B} \Omega^{2} \tilde{C} \xi=-\tilde{B}\left(C^{+} \ddot{y}_{\text {des }}+\Omega^{2} C^{+} y_{\text {des }}\right) \text {. }
$$

If $C B$ is invertible, then $\tilde{B} \widetilde{C}$ is also invertible and the dynamics of $\xi$ is governed by

$$
\ddot{\xi}+\Lambda \xi=L \rho
$$

where $\rho=\left[\begin{array}{ll}y_{\text {des }} & \ddot{y}_{\text {des }}\end{array}\right]^{T}$. We shall assume that $\operatorname{det}\left(s^{2} I+\Lambda\right)$ does not contain any purely imaginary roots.

If $C B$ is singular, then part of $\xi$ can be determined algebraically, and the rest is governed by a dynamic equation of the same form as (84). For the ease of presentation, but without loss of generality, we shall assume $C B$ is invertible.

For implementability, the initial condition, $(\xi(0), \dot{\xi}(0))$, needs to be chosen so that $\xi(t)$ that solves $(84)$ is uniformly bounded for all $t$. There are two equiralent approaches to find 
the initial condition. A Laplace transform approach was stated in [35] and a time domain approach in [9]. We will discuss both approaches here.

In the first approach, the Laplace transform of (84) is taken:

$$
\hat{\xi}(s)=\left(s^{2} I+\Lambda\right)^{-1}(L \hat{\rho}(s)+s \xi(0)+\dot{\xi}(0)) .
$$

Suppose $\hat{\rho}(s)$ is analytic in the open right half plane and has only simple poles on the imaginary axis (i.e., $\bar{y}_{\text {des }}(t)$ is uniformly bounded), then the terms in $\hat{\xi}(s)$ that can lead to unbounded time response are only those associated with the unstable roots of $\operatorname{det}\left(s^{2} I+\Lambda\right)$. Since $\Lambda$ is $n-m \times n-m$, there can be at most $n-m$ unstable roots. Correspondingly, there are $n-m$ residue vectors (in $\mathbf{R}^{m}$ ) which, when the contributions in $u f f$ are all set to zero, lead to $m(n-m)$ equations. There are $2(n-m)$ constants that we can choose in $(\xi(0), \dot{\xi}(0))$. Hence, if $m=2$, an initial condition can be chosen in general to nullify the residues associated with the unstable poles. If $m=1$, all residues can be nullified, implying the time response of $\xi(t)$ is zero after some finite $t$. The requirement that $m \leq 2$ appears to be unnecessarily strong as will be evident from the time domain analysis below.

An equivalent time domain approach can also be taken. First write (84) in the first order form:

$$
\left[\begin{array}{c}
\dot{\xi} \\
\vec{\xi}
\end{array}\right]=\left[\begin{array}{cc}
0 & I \\
-\Lambda & 0
\end{array}\right]\left[\begin{array}{l}
\xi \\
\dot{\xi}
\end{array}\right]+\left[\begin{array}{l}
0 \\
L
\end{array}\right] \rho .
$$

After transforming the coordinate according to the stable and unstable eigenspaces (recall that the center subspace is assumed to be trivial), the system is partitioned as

$$
\left[\begin{array}{c}
\dot{\gamma}_{+} \\
\dot{\gamma}_{-}
\end{array}\right]=\left[\begin{array}{cc}
\Lambda_{+} & 0 \\
0 & -\Lambda_{-}
\end{array}\right]\left[\begin{array}{l}
\gamma_{+} \\
\gamma_{-}
\end{array}\right]+\left[\begin{array}{c}
L_{+} \\
L_{-}
\end{array}\right] \rho
$$

where $\Lambda_{+}$and $\Lambda_{-}$are both strictly unstable. The unstable response is given by

$$
\begin{aligned}
\gamma_{+}(t) & =e^{\Lambda_{+} t} \gamma_{+}(0)+\int_{0}^{t} e^{\Lambda_{+}(t-\tau)} L_{+} \rho(\tau) d \tau \\
& =e^{\Lambda_{+} t}\left(\gamma_{+}(0)+\int_{0}^{t} e^{-\Lambda_{+} \tau} L_{+} \rho(\tau) d \tau\right)
\end{aligned}
$$

Choose

$$
\gamma_{+}(0)=-\int_{0}^{\infty} e^{-\Lambda_{+} \tau} L_{+} \rho(\tau) d \tau
$$

assuming the integral exists (which is true if $\ddot{y}_{\text {des }}$ is uniformly bounded). Then

$$
\begin{aligned}
\gamma_{+}(t) & =-\int_{t}^{\infty} e^{\Lambda_{+}(t-r)} L_{+} \rho(\tau) d \tau \\
& =-\int_{0}^{\infty} e^{-\Lambda_{+} s} L_{+} \rho(t+s) d s
\end{aligned}
$$

The uniform boundedness of $\gamma_{+}$follows from the strict stability of $-\Lambda_{+}$:

$$
\left\|\gamma_{+}(t)\right\| \leq \int_{0}^{\infty} M e^{-\sigma_{+} s}\left\|L_{+}\right\|\|\rho\|_{L_{\infty}} d s=\frac{M}{\sigma_{+}}\left\|L_{+}\right\|\|\rho\|_{L_{\infty}}
$$


where $-\sigma_{+}=\max _{i} \operatorname{Re} \lambda_{i}\left(-\Lambda_{+}\right), \lambda_{i}$ denotes the $i^{\text {th }}$ eigenvalue, and $M$ is some positive constant. In [35], it was pointed out that the procedure of choosing the initial condition to guarantee the boundedness of $u f f$ is highly sensitive numerically since any slight numerical error could lead to divergence. Eq. (87) shows that $\gamma_{+}(t)$ can be stably computed even though $\Lambda_{+}$is strictly unstable. Note that the condition on the number of input/output pairs is no longer required in this analysis. This discrepancy appears to be due to some relationship in the residues that we are not taking advantage of.

As in the Laplace transform approach, $\gamma_{-}(0)$ can be chosen to achieve the zero steady state for $\gamma_{-}$if $\int_{0}^{\infty} e^{\Lambda_{-} \tau} L_{-} \rho(\tau) d \tau<\infty$.

To illustrate the procedure described above, consider a simple example presented in [35]:

$$
\begin{gathered}
\ddot{q}+\left[\begin{array}{cc}
1 & -1 \\
-1 & 1
\end{array}\right] q=\left[\begin{array}{c}
\frac{2}{3} \\
-\frac{1}{3}
\end{array}\right] u \\
y=\left[\begin{array}{ll}
0 & 1
\end{array}\right] q .
\end{gathered}
$$

Follow the procedure outline before, $q_{d e s}$ can be represented as

$$
q_{\text {des }}=\left[\begin{array}{l}
0 \\
1
\end{array}\right] y_{\text {des }}+\left[\begin{array}{l}
1 \\
0
\end{array}\right] \xi
$$

After substitution into the dynamic equation, $u_{f f}$ can be solved:

$$
u_{f f}=\frac{3}{5}\left(-\ddot{y}_{\text {des }}-3 y_{\text {des }}+2 \vec{\xi}+3 \xi\right)
$$

Substitute back into the dynamic equation for $\xi$, we obtain:

$$
\ddot{\xi}-\xi=-y_{\text {des }}-2 \ddot{y}_{\text {des }} \text {. }
$$

Suppose the desired output trajectory is given by

$$
\ddot{y}_{\text {des }}= \begin{cases}1 & 0 \leq t<1 \\ -1 & 1 \leq t<2 \\ 0 & t \geq 2\end{cases}
$$

and $y_{\text {des }}(0)=\dot{y}_{\text {des }}(0)=0$. For simplicity, make a change of variable $\eta=\xi-y_{\text {des }}$, then

$$
\begin{aligned}
\ddot{\eta}-\eta & =-3 \ddot{y}_{\text {des }} \\
u_{f f} & =\frac{3}{5}\left(2 \ddot{\eta}+3 \eta+3 \ddot{y}_{\text {des }}\right)
\end{aligned}
$$

The Laplace transform of $\ddot{y}_{\text {des }}$ is

$$
\widehat{y_{d e s}}(s)=\frac{\left(1-e^{-s}\right)^{2}}{s}
$$

Therefore,

$$
\hat{\eta}(s)(s)=\frac{\left(-3 s^{-1}\left(1-e^{-s}\right)^{2}+s \eta(0)+\dot{\eta}(0)\right)}{s^{2}-1} .
$$


If only the unstable residue is to be canceled as suggested in [35], one choice for the initial condition is

$$
\eta(0)=\dot{\eta}(0)=\frac{3}{2}\left(1-e^{-1}\right)^{2} .
$$

In this case, since $m=1$, residues associated with both poles can in fact be canceled by choosing

$$
\left[\begin{array}{l}
\eta(0) \\
\dot{\eta}(0)
\end{array}\right]=\left[\begin{array}{c}
\frac{3}{2}\left(\left(1-e^{-1}\right)^{2}+(1-e)^{2}\right) \\
-\frac{3}{2}\left(-\left(1-e^{-1}\right)^{2}+(1-e)^{2}\right)
\end{array}\right] \text {. }
$$

An equivalent time domain approach can also be taken. The solution of (88) is

$$
\eta(t)=\left[\begin{array}{cc}
\cosh t & \sinh t \\
\sinh t & \cosh t
\end{array}\right]\left(\left[\begin{array}{l}
\eta(0) \\
\dot{\eta}(0)
\end{array}\right]-3 \int_{0}^{t}\left[\begin{array}{cc}
\cosh \tau & -\sinh \tau \\
-\sinh \tau & \cosh \tau
\end{array}\right]\left[\begin{array}{l}
0 \\
1
\end{array}\right] \ddot{y}_{\text {des }}(\tau) d \tau\right) .
$$

After using (89), the integral, for $t \geq 2$, is a constant:

$$
\int_{0}^{t}\left[\begin{array}{cc}
\cosh \tau & -\sinh \tau \\
-\sinh \tau & \cosh \tau
\end{array}\right]\left[\begin{array}{l}
0 \\
1
\end{array}\right] \ddot{y}_{d e s}(\tau) d \tau=\left[\begin{array}{c}
1-2 \cosh 1+\cosh 2 \\
2 \sinh 1-\sinh 2
\end{array}\right] \text {. }
$$

Since the stable eigenspace is spanned by $\left[\begin{array}{c}1 \\ -1\end{array}\right]$ and the unstable eigenspace is spanned by $\left[\begin{array}{l}1 \\ 1\end{array}\right]$, choosing the initial condition according to (92) leads to

$$
\eta(t)=-\frac{3}{2} e^{-t}\left(1+e^{2}-2 e\right)
$$

for $t \geq 2$ and choosing the initial condition according to (93) leads to $\eta(t)=0$ for $t \geq 2$. Clearly, the latter choice has the advantage that the feedforward control (cf. (91)) is no longer needed after the desired output trajectory has come to a rest.

When the desired output is assumed generated from a reference model and the model and plant parameters satisfy a model matching condition, a solution of (79)-(80) can be more easily solved. This is called the regulator approach, a version of which, called the command generator tracker theory, was proposed in [7]. The nonlinear version can be found in [36]. Application to the flexible arm control can be found in [37]. We present this approach for a general linear time invariant system. Consider

$$
\begin{aligned}
& \dot{x}_{\text {des }}=A x_{\text {des }}+B u_{f f} \\
& y_{\text {des }}=C x_{\text {des }} .
\end{aligned}
$$

The desired output $y_{d e s}$ is generated from a linear time invariant reference model:

$$
\begin{aligned}
\dot{w} & =S w \\
y_{\text {des }} & =Q w
\end{aligned}
$$

where $w \in \mathbf{R}^{k}$. We seek a solution of the form

$$
u_{f f}=F w
$$


where $F$ and a matrix $P$ together should satisfy

$$
\begin{aligned}
P S-A P & =B F \\
C P & =Q
\end{aligned}
$$

which are called the model matching conditions. The initial condition $x_{\text {des }}(0)$ should be chosen as

$$
x_{\text {des }}(0)=P w(0) .
$$

Clearly, if the exosystem is stable, the feedforward signal will be uniformly bounded.

The model matching condition (95) can be written as a linear matrix equation by using Kronecker products [38]:

$$
\left[\begin{array}{cc}
S^{T} \otimes I-I \otimes A & I \otimes B \\
I \otimes C & 0
\end{array}\right]\left[\begin{array}{c}
P_{c} \\
F_{c}
\end{array}\right]=\left[\begin{array}{c}
0 \\
Q_{c}
\end{array}\right]
$$

where the subscript $c$ denotes the vector formed by stacking up the columns in the matrix. For a given plant and exosystem, the solvability of (98) can be readily checked (a sufficient condition is the invertibility of the matrix containing $A, B, C$, and $S$ ), and if solvable, $P$ and $F$ can also be easily found.

The feedforward $u_{f f}$ given by the linear regulator approach is a particular solution of (82) from the plant inversion. It would be interesting to query if the initial condition chosen as in (97) is related to the initial condition chosen based on the plant inversion approach described earlier (either through the Laplace transformation or time domain solution). In Appendix $A$, it was shown for a single flexible link tracking a sinusoid, that the initial condition from the regulator approach is the same as the one chosen to cancel all of the residues. We are currently seeking the generalization of this result.

\section{Adaptive Control}

The feedforward control in either set point or tracking case requires a great deal of model information. It is highly desirable to adaptively update this signal without requiring explicit knowledge of the plant parameters. To this end, consider the closed loop system as an internally asymptotically stable system driven by the input $u_{f f}$. Based on our passivity approach, the closed loop system is passive but in general not strictly passive. Our basic idea is to choose a new output such that the I/O pair between the input $u_{f f}$ and this output is strictly passive. Then any passive adaptation for $u_{f f}$ can be used to preserve the state asymptotic stability.

We will only consider the linearized closed loop plant here, the full nonlinear version is under development. Suppose that the linearized closed loop plant is of the form

$$
\dot{x}=A x+B\left(u-u_{f f}\right)
$$

where $A$ is exponentially stable, $u_{f f}$ is the unknown desired feedforward, and $u$ is the adaptive feedforward. By the Lyapunov's theorem [39], for any $Q>0$, there exists $P>0$ such that

$$
A^{T} P+P . A=-Q \text {. }
$$


Now define $C=B^{T} P$ as the new output map. Then the triplet $(A, B, C)$ is strictly positive real [40]. The adaptation for $u_{f f}$ is now straightforward. Using the standard linear-inparameter formulation [41], suppose $u_{f f}$ can be parameterized as

$$
{ }^{u} f f=H \lambda
$$

where $H$ in the known regressor matrix and $\lambda$ is the unknown parameter vector. For the set point control case, $H=I$ and $\lambda$ is a constant vector. For the regulator approach, $H$ contains $w$ (state of the exosystem) and $\lambda$ consists of columns of $F$ (cf. (94)). For the tracking of a general desired output, $H$ depends on $\theta, \dot{\theta}, y_{\text {des }}$ and its higher derivatives. In this case, finding the structure of $H$ itself may be difficult. A viable approach may be to approximate $H$ by some expansion and slowly adapt the approximation. The neural net approach in [42] is a possibility that we shall explore.

To derive the adaptation rule, consider the Lyapunov function candidate

$$
V=x^{T} P x+\Delta \lambda^{T} \Gamma^{-1} \Delta \lambda
$$

where $\Delta \lambda=\hat{\lambda}-\lambda, \hat{\lambda}$ is the estimate of the unknown vector $\lambda$. Since the output is chosen so the system is strictly positive real, the derivative along the solution becomes

$$
\dot{V}=-x^{T} Q x+2\left(u-u_{f f}\right)^{T} y+2 \Delta \lambda^{T} \Gamma^{-1} \dot{\hat{\lambda}} .
$$

Choose the adaptive feedforward based on the estimated parameter:

$$
u=\hat{u}_{f f}=H \hat{\lambda}
$$

Then

$$
\dot{V}=-x^{T} Q x+2 \Delta \lambda^{T}\left(H^{T} y+\Gamma^{-1} \dot{\hat{\lambda}}\right) .
$$

Hence, with the following gradient update rule for $\hat{\lambda}$ :

$$
\dot{\hat{\lambda}}=-\Gamma H^{T} y
$$

$\dot{V}$ is negative semidefinite. This implies that all states and parameter estimate error are bounded, and furthermore, by Barbalat's Lemma [43], $x$ converges to zero asymptotically. For the set point control case, the adaptive parameter update simply reduces to the integral control law.

We are currently extending this argument to the nonlinear systems by using the nonlinear Lyapunov equation.

\section{Simulation Results}

To illustrate the results shown in this paper, we consider a simple one flexible joint robot. This model is taken from [44]. The dynamical equation is given by

$$
\begin{aligned}
\frac{1}{3} m \ell^{2} \ddot{\theta}_{\ell}+B_{\ell} \dot{\theta}_{\ell}+\frac{m g \ell}{2} \sin \theta_{\ell}+k\left(\theta_{\ell}-\frac{1}{n} \theta_{m}\right) & =0 \\
J_{m} \ddot{\theta}_{m}+B_{m} \dot{\theta}_{m}-\frac{k}{n}\left(\theta_{\ell}-\frac{1}{n} \theta_{m}\right) & =u .
\end{aligned}
$$




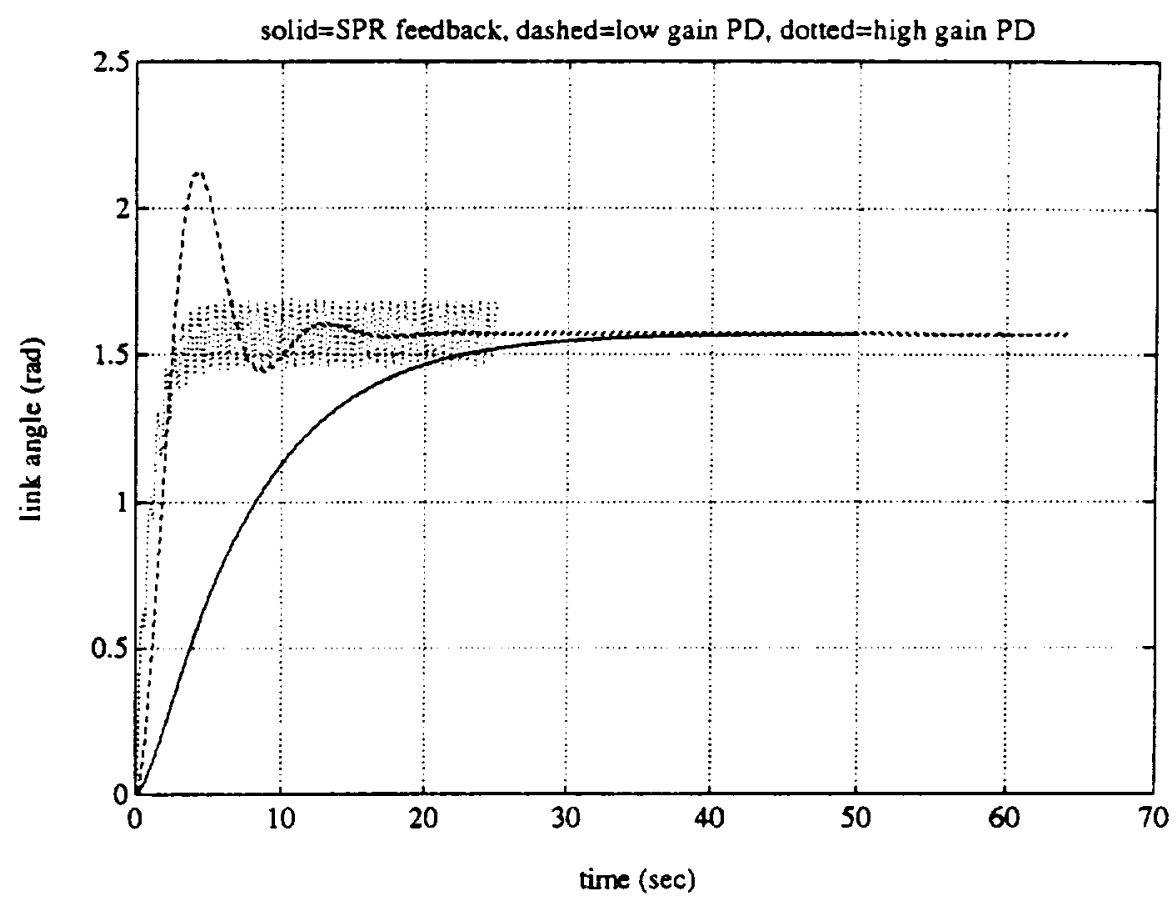

Figure 1: Comparison between Link Angle Step Responses

Numerical values used are (all quantities are in SI units):

$$
m=10, \quad g=9.8, \quad \ell=.5, \quad k=100, \quad n=50, \quad J_{m}=.08 .
$$

The damping factors are assumed to be zero except for one of the tracking case.

Fig. 1 and 2 show the step response $\left(90^{\circ}\right.$ commanded link angle step) of the link angle and angular velocity under high gain $\mathrm{PD}\left(k_{p}=k_{v}=5\right)$, low gain $\mathrm{PD}\left(k_{p}=k_{v}=.05\right)$, and SPR filter feedback. From these plots, it is evident that under high gain PD control the response of the closed-loop system, though stable, is highly oscillatory. Lower gain improves the overshoot and reduces the oscillation, but the best case corresponds to a fourth order positive real (PR) bandpass filter in parallel to the low gain PD, where the passband is roughly at the natural frequency of the spring. For this case, the transient is further slowed, but the residue oscillation is reduced by about $50 \%$.

The PR filter transfer function is given by $\frac{b(s)}{a(s)}$ where

$$
\begin{aligned}
& a(s)=s^{4}+75 s^{3}+1925 s^{2}+19125 s+56250 \quad \text { roots at }\{-30,-25,-15,-5\} \\
& b(s)=100\left(s^{3}+31 s^{2}+230 s+200\right) \quad \text { roots at }\{-20,-10,-1\}
\end{aligned}
$$

The phase and magnitude plots of this filter are shown in Fig. 3. It should be emphasized that virtually no tuning of this filter was carried out to optimize the performance. It was chosen basically to have a peak roughly at the resonant frequency of the linearized system.

Fig. 4 shows the tracking of a cycloid over the same range. Since the desired trajectory eventually reaches a steady state, the set point control analysis can be applied. Fig. 5 shows the tracking of a slowly varying sinusoidal signal. By the analysis in section 7.0 .2 , it is not surprising that the actual and desired outputs, $\theta_{\ell}$ and $\theta_{\ell_{\text {dee }}}$, are virtually undistinguishable. When the desired sinusoid becomes too fast as in Fig. 6 (same as the one used in [44]). a significant error develops. This is as expected as the system remains $1 / 0$ stable but no 


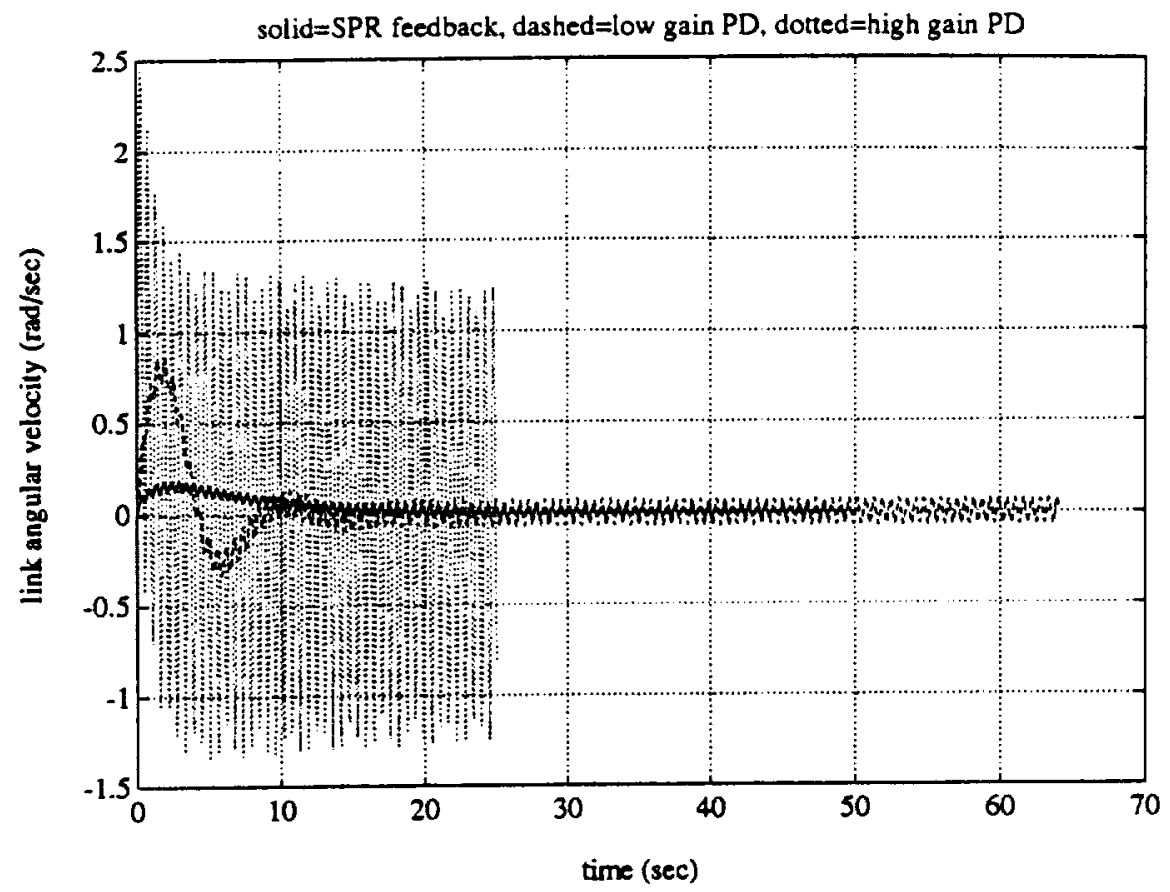

Figure 2: Comparison between Link Angular Velocity Step Responses
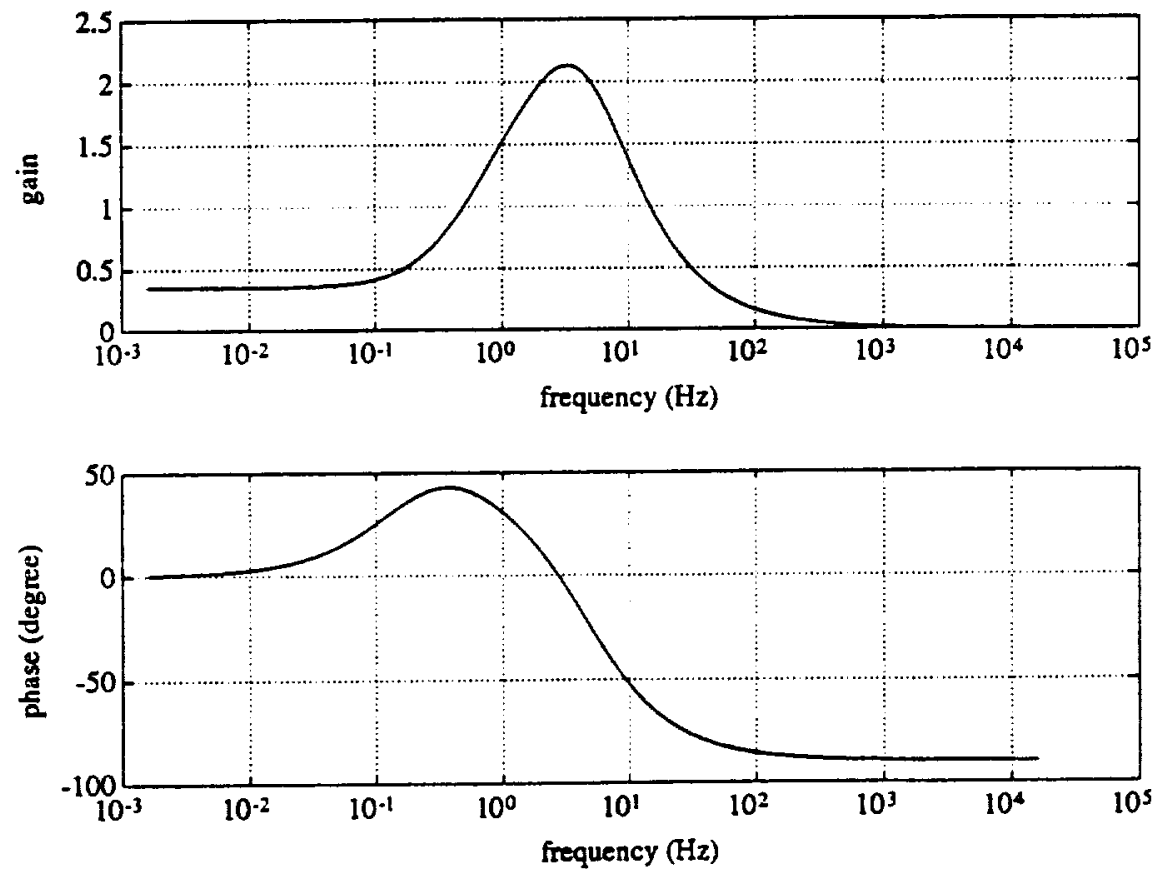

Figure 3: Magnitude and Phase Plot of the Positive Real Filter 


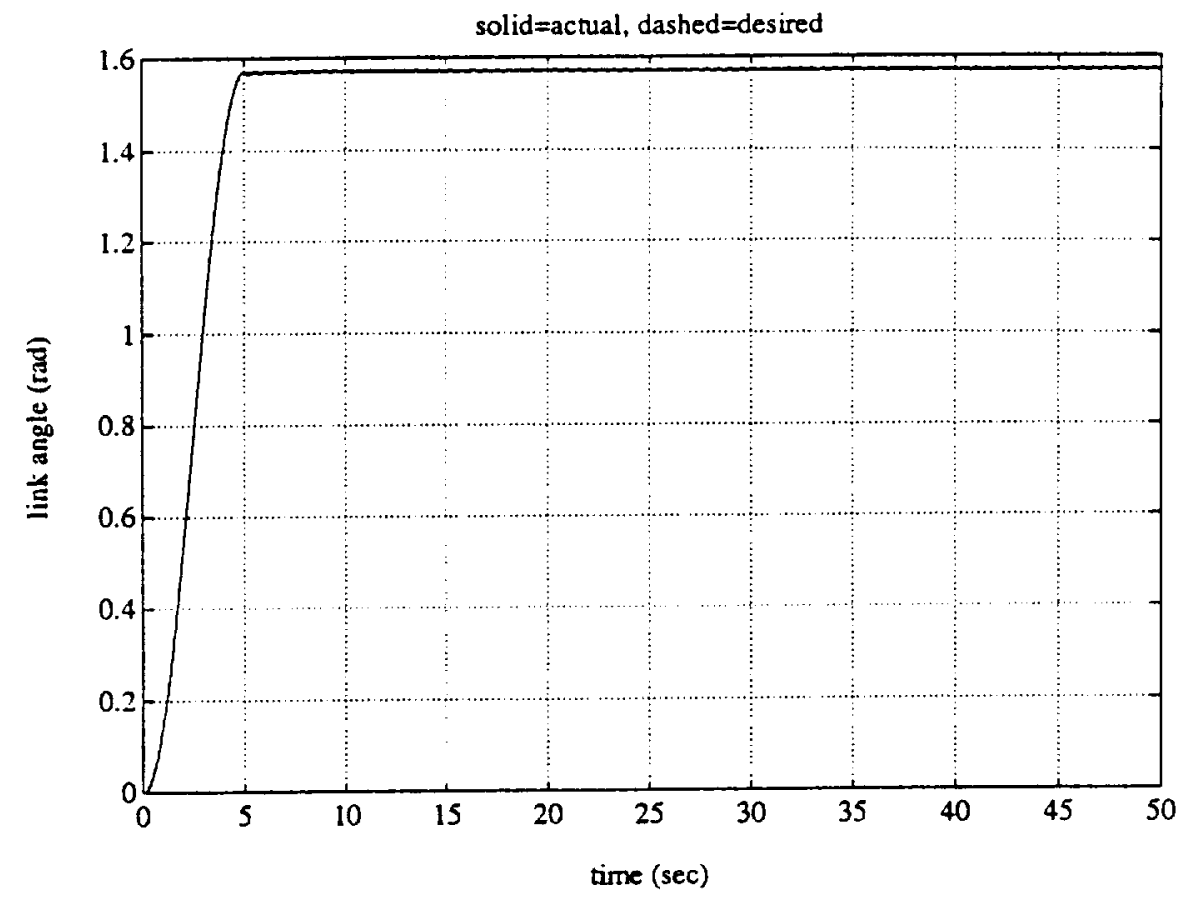

Figure 4: Set Point Control with Transient Shaping

longer internally asymptotically stable. It should be noted that even in this case, the PR filter feedback has much less (by about 50\%) error than the constant gain PD feedback.

When the link damping is included, $B_{\ell}=36$ as in [44] (but not motor damping), so the analysis in section 7.0 .3 can be applied, faithful tracking is once again attained; the results are shown in Fig. 7.

In all these cases, the same SPR filter as in the set point control case is used, except the feedback signal is now $\dot{\theta}_{m}-\dot{\theta}_{m_{\text {dee }}}$ instead of $\dot{\theta}_{m}$. These examples demonstrate, in a very preliminary sense, the possibility of tuning the SPR filter to attain an improved performance in terms of step response and the efficacy of using the feedforward for trajectory tracking.

\section{Conclusions}

In this paper, we derived a large class of asymptotically stabilizing control laws for a general class of mechanical systems typified by structures containing uncontrolled degrees of freedom which are elastically coupled to the controlled degrees of freedom. The idea is to first choose a feedforward to form an error system. Next a position loop is closed to obtain passivity, stabilizability and detectability for a particular input/output pair. Then any strictly passive output feedback can be used to achieve closed loop stability. Through a simple example, it is shown that the dynamic nature of the feedback can be exploited to enhance performance. A systematic procedure for designing the feedback system for performance enhancement and adaptive update of the feedforward are currently under investigation. 


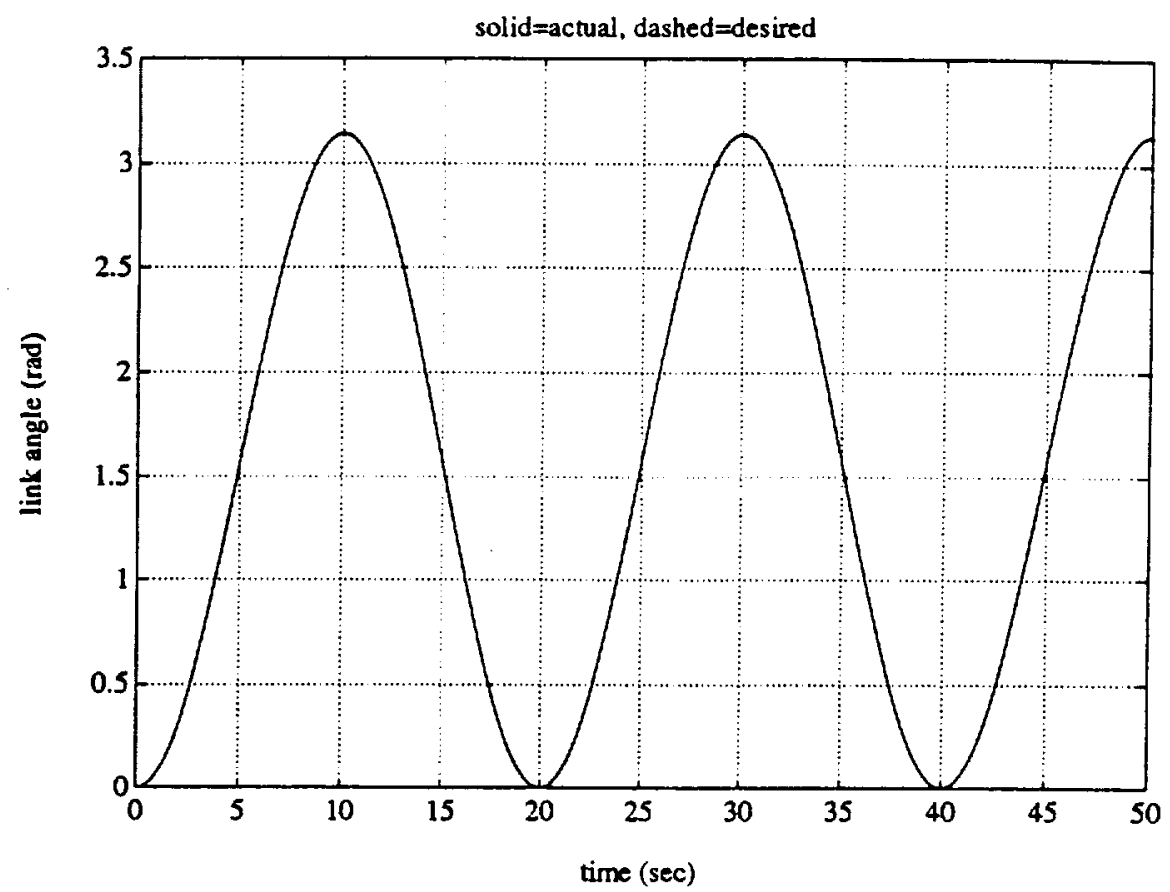

Figure 5: Slow Sinusoid Tracking Control

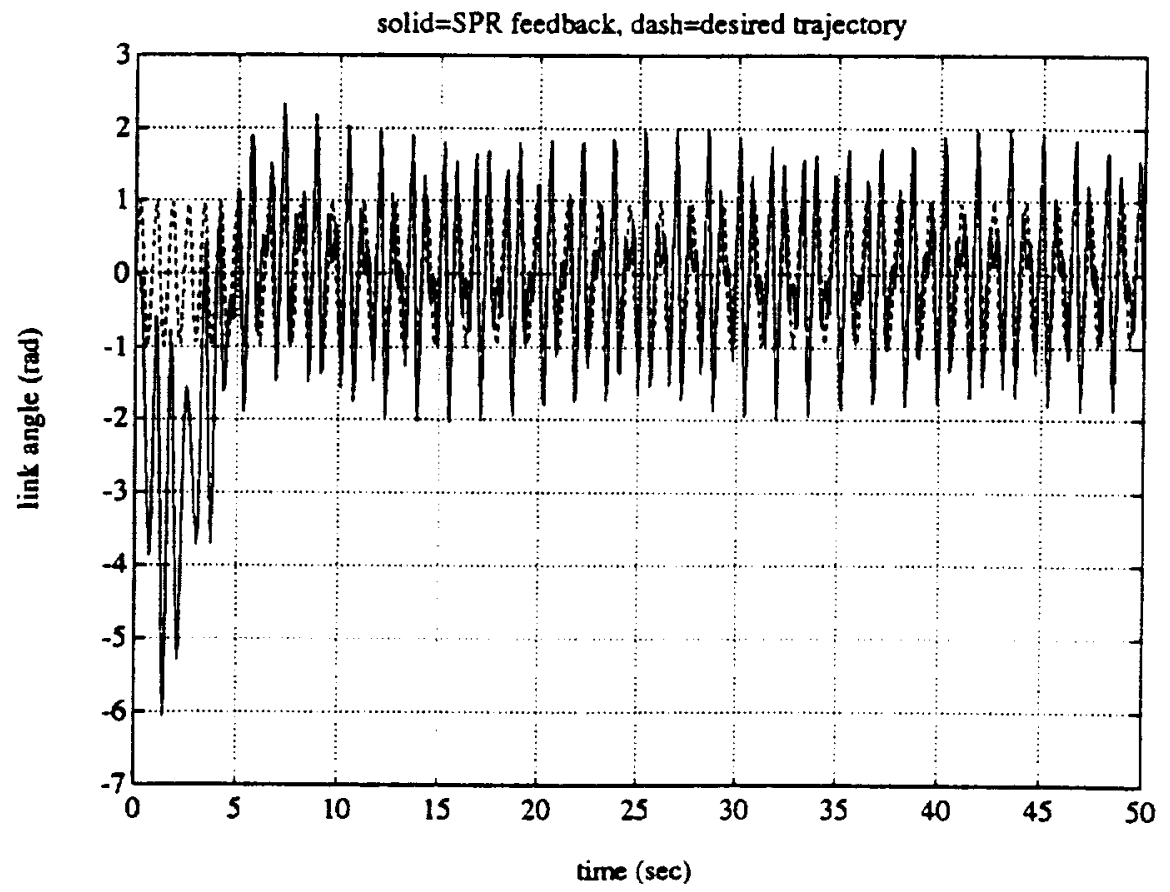

Figure 6: Fast Sinusoid Tracking Control: Zero Damping Case 


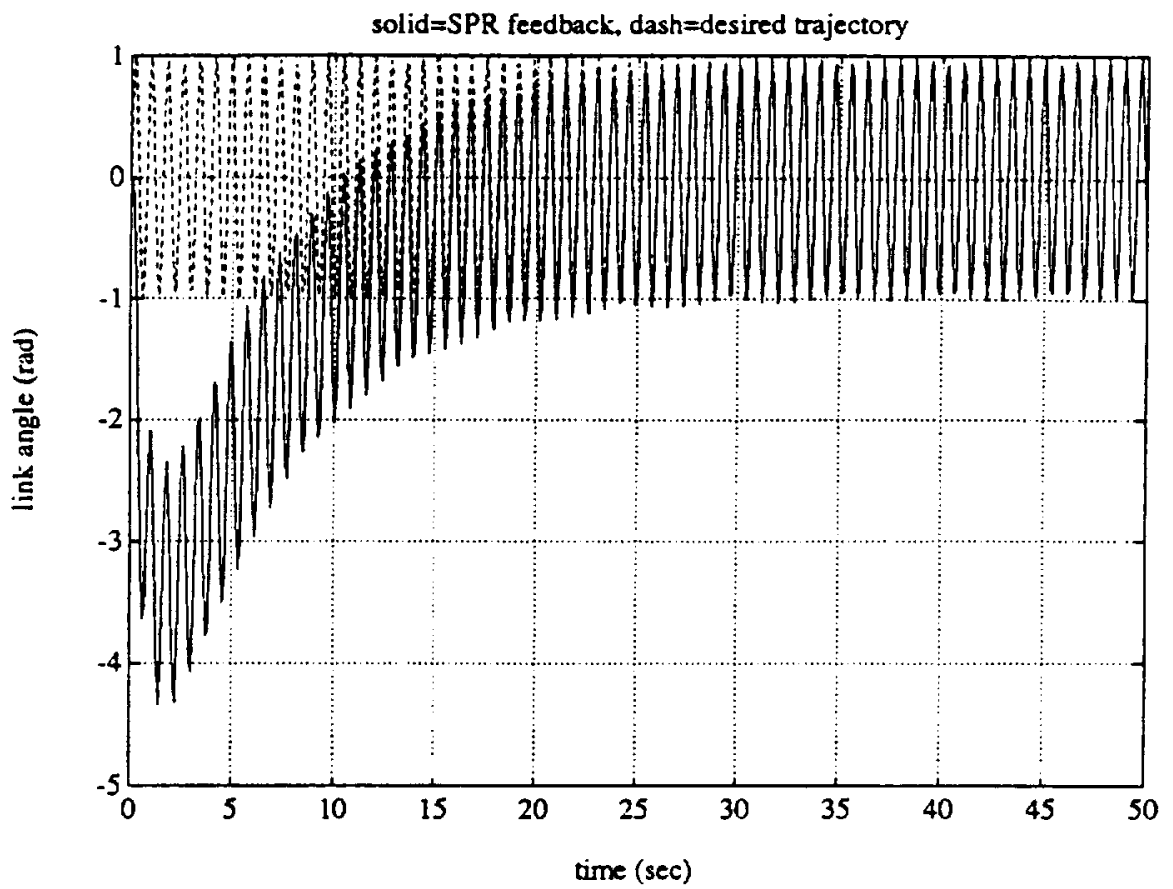

Figure 7: Fast Sinusoid Tracking Control: Nonzero Link Damping Case

\section{Acknowledgment}

This research has been conducted while the first author was a visiting scholar at CIRSSE, Rensselaer Polytechnic Institute, Troy, NY, whose local support is gratefully acknowledged. The second author was supported in part by the Center for Intelligent Robotic Systems for Space Explorations at the Rensselaer Polytechnic Institute under grant NAGW 1333, NASA Johnson Space Center under grant No.NAG 9-462/Basic and National Science Foundation under Grant No.MSS-8910437.

\section{References}

[1] L. Meirovith. Analytic Methods in Vibration. The MacMillan Company, New York, 1967.

[2] M. Takegaki and S. Arimoto. A new feedback method for dynamic control of manipulators. ASME J. Dynamic Systems, Measurement and Control, 102, June 1981.

[3] S. Arimoto and F. Miyazaki. Stability and robustness of pd feedback control with gravity compensation for robot manipulator. In ASME Winter Meeting, pages 6i-i2, Anaheim, CA, December 1986.

[4] P. Tomei. Point-to-point control of elastic joint robots. In Proc. Int. Symp. on Intelligent Robotics, Bangalore, India, January 1991.

[5] H.G. Lee. H. Kanoh. S. Kawamura, F. Miyazaki, and S. Arimoto. Stability analysis of a one-link flexible arm control by a linear feedback law. In S.G. Tzafestas T. Futagami 
and Y. Sunahara, editors, Distributed Parameter Systems: Modelling and Simulation, pages 345-352. Elsevier Science Publishers B.V. (North-Holland), 1989.

[6] M.W. Spong. Control of flexible joint robots: A survey. UILU-ENG-90-2203 DC-116, University of Illinois at Urbana-Champaign, February 1990.

[7] R.J. Benhabib, R.P. Iwens, and R.L. Jackson. Stability of distributed control for large Hexible structures using positivity concepts. In AIAA Guidance and Control Conference, Paper No. 79-1780, Boulder, Co., August 1979.

[8] E. Bayo. Computed torque for the position control of open-chain flexible robots. In Proc. 1988 IEEE Robotics and Automation Conference, pages 316-321, Philadelphia, PA, April 1988.

[9] D.S. Kwon and W.J. Book. An inverse dynamic method yielding flexible manipulator state trajectories. In Proc. 1990 American Control Conference, pages 186-193, San Diego, CA, May 1990.

[10] B. Paden and B. Riedle. A positive-real modification of a class of nonlinear controllers for robot manipulators. In Proc. 1988 American Control Conference, Atlanta, GA, June 1988.

[11] B. Paden, B. Riedle, and E. Bayo. Exponentially stable tracking control for multi-joint flexible-link manipulators. In Proc. 1990 American Control Conference, pages 680-684, San Diego, CA, June 1990.

[12] J.C. Willems. Dissipative dynamical systems, part I: General theory, part II: Linear systems with quadratic supply rate. Arch. Rational Mech. Anal., 45:321-393, 1972.

[13] P.J. Moylan. Implication of passivity in a class of nonlinear systems. IEEE Transaction on Automatic Control, 19:373-381, 1974.

[14] G. Zames. On the input/output stability of time-varying nonlinear feedback systems, part I: Conditions derived using concepts of loop gain, conicity, and positivity, part II: Conditions involving circles in the frequency plane and sector nonlinearities. IEEE Transaction on Automatic Control, 11(3):465-476, 1966.

[15] D.J. Hill and P.J. Moylan. Dissipative dynamical systems: Basic input-output and state properties. J. Franklin Institute, 309(5):327-35i, 1980.

[16] C.I. Byrnes, A. Isidori, and J.C. Willems. Stabilization and output regulation of nonlinear systems in the large. In Proc. 29th IEEE Conf. Decision and Control, Honolulu, HI, December 1990.

[17] S.H. Murphy. Modeling and Simulation of Multiple Cooperating Manipulators on a Mobile Platform. PhD thesis, Rensselaer Polytechnic Institute, Troy, NY., Nov 1990.

[18] M.W. Spong. Modeling and control of elastic joint robots. ASME J. Dynamic Systems. .Wensurement and Control, 109:310-319, Dec. 198i. 
[19] A. De Luca. Dynamic control of robots with joint elasticity. In Proc. 1988 IEEE Robotics and Automation Conference, pages 152-158, Philadelphia, PA, 1988.

[20] S. Nicosia, F. Nicolò, and D. Lentini. Dynamical control of industrial robots with elastic and dissipative joints. In Proc. 8th IFAC World Congress, pages 1933-1939, Kyoto, 1981.

[21] T.M. Apostol. Mathematical Analysis. Addison-Wesley, 2 edition, 1975.

[22] D.E. Koditschek. Adaptive techniques for mechanical systems. In Proc. 5th Yale Workshop on Applications of Adaptive Systems Theory, pages 259-265, New Haven, CT, May 1987.

[23] J.-J. E. Slotine and W. Li. On the adaptive control of robot manipulators. In $A S M E$ Winter Meeting, pages 43-50, Anaheim, CA, 1986.

[24] J.T. Wen and D.S. Bayard. Simple robust control laws for robotic manipulators, part I: Non-adaptive case. In JPL/NASA Telerobotics Workshop, January 1987.

[25] R. Ortega and M.W. Spong. Adaptive motion control of rigid robots: A tutorial. In Proc. 27th IEEE Conf. Decision and Control, pages 1575-1584, Austin, TX, December 1988.

[26] S.H. Murphy, J.T. Wen, and G.N. Saridis. Efficient dynamic simulation of flexibly jointed manipulators. In Proc. 29th IEEE Conf. Decision and Control, pages 545-550, Honolulu, HI, December 1990.

[27] L. Lanari and J.T. Wen. A family of asymptotic stable control laws for flexible robots based on a passivity approach. CIRSSE Report 85, Rensselaer Polytechnic Institute. February 1991.

[28] C. Trudel. SRMS joint servo and gearbox math model. Technical Report SPAR-TM. 1213, Issue B, SPAR Aerospace, May 1977.

[29] C. DeSimone and Nicolo F. On the control of elastic robots by feedback decoupling. IEEE Joumal of Robotics and Automation, 1(2):64-69, 1986.

[30] F. Bellezza, L. Lanari, and G. Ulivi. Exact modeling of the flexible slewing link. In Proc. 1990 IEEE Robotics and A utomation Conference, pages $734-739$, Cincinnati, OH, 1990.

[31] F. Wang and J.T. Wen. Nonlinear dynamical model and control for a flexible beam. CIRSSE Report 75, Rensselaer Polytechnic Institute, November 1990.

[32] N. Sadegh and R. Horowitz. Stability and robustness analysis of a class of adaptive controllers for robotic manipulators. Int. J. Robotics Research. 1989.

[33] M. Vidyasagar. Vonlinear Systems Analysis. Prentice-Hall. ViJ. 197s. 
[34] J.T. Wen and D.S. Bayard. A new class of control laws for robotic manipulators, Part I: Non-adaptive case. Int. J. Control, 47(5):1361-1385, 1988.

[35] E. Bayo and H. Moulin. An efficient computation of the inverse dynamics of flexible manipulators in the time domain. In Proc. 1989 IEEE Robotics and Automation Conference, Scottsdale, AZ, 1989.

[36] A. Isidori. Nonlinear Control Systems. Springer-Verlag, second edition, 1989.

[37] A. De Luca, L. Lanari, and G. Ulivi. Output regulation of a flexible robot arm. In 9th INRIA Int. Conf. on Analysis and Optimization, pages 833-842, Antibes, France, June 1990.

[38] J.W. Brewer. Kronecker products in systems theory. IEEE Trans. on Circuits and Systems, 25:772-781, September 1978.

[39] W.M. Wonham. Linear Multivariable Control: A Geometric Approach. Springer-Verlag, New York, 1979.

[40] J.T. Wen. Time domain and frequency domain conditions for strict positive realness. IEEE Trans. on Automatic Control, 33(10), Oct 1988.

[41] P. Khosla and T. Kanade. Parameter identification of robot dynamics. In Proc. 25th IEEE Conf. Decision and Control, Fort Lauderdale, FL, December 1985.

[42] W. Cheng and J. Wen. A two-time-scale neural tracking controller for a class of nonlinear systems. Ral report, Rensselaer Polytechnic Institute, May 1991.

[43] V.M. Popov. Hyperstability of Control Systems. Springer-Verlag, New York, 1973.

[44] M.W. Spong, K. Khorasani, and P.V. Kokotovic. An integral manifold approach to the feedback control of flexible joint robots. IEEE J. of Robotics and Automation, RA-3(4):291-300, August 1987.

\section{Appendix A}

Consider the linear model for a flexible link, where, for simplicity, only one flexible mode is included:

$$
\begin{aligned}
\ddot{q}+\Omega^{2} q & =B u \\
C q & =y
\end{aligned}
$$

with

$$
\Omega^{2}=\left[\begin{array}{cc}
0 & 0 \\
0 & \omega^{2}
\end{array}\right], \quad B=\left[\begin{array}{l}
\psi_{0}^{\prime}(0) \\
\psi_{1}^{\prime}(0)
\end{array}\right], \quad \mathbf{q}(t)=\left[\begin{array}{l}
\theta(t) \\
\delta(t)
\end{array}\right] .
$$


Variable $\theta(t)$ denotes the rigid body displacement, $\delta(t)$ denotes the flexible modal displacement, $\psi_{1}(x)$ is the eigenfunction associated with the flexible mode and 'indicates the spatial derivative.

For the output trajectory tracking problem, the desired output trajectory $y_{\text {des }}(t)$ is given and the objective of the steady-state analysis is to find a desired state $q_{\text {des }}(t)$ and feedforward $u_{f}(t)$ such that

$$
\begin{aligned}
q_{\text {des }}+\Omega^{2} q_{\text {des }} & =B u_{f f} \\
C q_{\text {des }} & =y_{\text {des }} .
\end{aligned}
$$

Consider the tip angular position as the output variable:

$$
y=\left[\begin{array}{ll}
1 & \frac{\psi_{1}(\ell)}{\ell}
\end{array}\right] \mathrm{q}=C \mathrm{q}
$$

For this case study, the desired output trajectory is chosen to be a sinusoid $y_{\text {des }}(t)=\sin \left(\omega_{\tau} t\right)$. For later use, we also define the following two matrices

$$
C^{+}=\frac{1}{1+\psi_{\ell}^{2}}\left[\begin{array}{c}
1 \\
\psi_{\ell}
\end{array}\right], \quad \tilde{C}=\left[\begin{array}{c}
-\psi_{\ell} \\
1
\end{array}\right], \quad \text { where } \psi_{\ell}=\frac{\psi_{1}(\ell)}{\ell}
$$

The purpose of this appendix is to derive $u_{f f}$ using three different methods: regulation, Laplace transform, and time domain. We will show that all three methods lead to the same solution.

\section{Regulator method}

The desired output trajectory (sinusoid) can be generated by the following exosystem

$$
\dot{w}=S w=\left[\begin{array}{cc}
0 & \omega_{r} \\
-\omega_{r} & 0
\end{array}\right]\left[\begin{array}{l}
w_{1} \\
w_{2}
\end{array}\right], \quad w(0)=\left[\begin{array}{l}
0 \\
1
\end{array}\right], \quad y_{d e s}=Q w=\left[\begin{array}{ll}
1 & 0
\end{array}\right] w
$$

The solution to the linear regulator problem requires the following matrix equations to be solved in $P$ and $F$.

$$
\begin{aligned}
P S & =A P+B F \\
Q & =C P .
\end{aligned}
$$

Equations (111) can be easily solved:

$$
\begin{aligned}
P_{11} & =\frac{\left(\omega_{r}^{2}-\omega^{2}\right) / N}{\left(\omega_{r}^{2}-\omega^{2}\right) / N+\frac{\omega^{\prime}(0)}{\psi_{0}^{\prime}(0)} \omega_{r}^{2}} & P_{12} & =0 \\
P_{21} & =\frac{1-P_{11}}{\psi_{l}} & P_{22} & =0 \\
P_{31} & =0, & P_{32} & =\omega_{r} P_{11} \\
P_{41} & =0, & P_{42} & =\omega_{r} P_{21} \\
F_{1} & =-\frac{\omega_{r}^{2}}{\psi_{0}^{\prime}(0)} P_{11} . & F_{2} & =0 .
\end{aligned}
$$


Therefore, the required solution is

$$
\begin{aligned}
q_{\text {des }}(0) & =\left[\begin{array}{c}
0 \\
0 \\
P_{32} \\
P_{42}
\end{array}\right] \\
u_{f f}(t) & =F_{1} w_{1}(t)=F_{1} y_{\text {des }}(t) .
\end{aligned}
$$

\section{Laplace transform method}

Following the procedure outlined in section 7.0 .4 one obtains the following equation

$$
\hat{\xi}(s)=\left(s^{2} I+\Lambda\right)^{-1}(L \hat{\rho}(s)+s \xi(0)+\dot{\xi}(0))
$$

with

$$
\Lambda=\frac{\omega^{2}}{1+\psi_{\ell} \psi_{1}^{\prime}(0) / \psi_{0}^{\prime}(0)}, \quad L=\frac{-\left(\psi_{\ell} \omega^{2} \hat{y}(s)+\left(\psi_{\ell}-\psi_{1}^{\prime}(0) / \psi_{0}^{\prime}(0)\right) \hat{\ddot{y}}(s)\right)}{\left(1+\psi_{\ell} \psi_{1}^{\prime}(0) / \psi_{0}^{\prime}(0)\right)\left(1+\psi_{\ell}^{2}\right)}
$$

For simplicity $\hat{y}(s)$ and $\hat{\hat{y}}(s)$ denote the Laplace transorm of the desired output trajectory and acceleration respectively. The $\hat{\xi}(s)$ has an unstable mode iff

$$
\left(1+\psi_{\ell} \psi_{1}^{\prime}(0) / \psi_{0}^{\prime}(0)\right)<0
$$

which corresponds to the condition for which unstable zero-dynamics exists (non minimum phase condition). For simplicity write

$$
\Psi^{2}=-\left(1+\psi_{\ell} \psi_{1}^{\prime}(0) / \psi_{0}^{\prime}(0)\right), \quad \bar{\omega}=\frac{\omega}{\Psi}, \quad \text { with } \quad \Psi>0
$$

so that the poles can be written as

$$
s_{\text {stable }}=-\bar{\omega}, \quad s_{\text {unstable }}=\bar{\omega},
$$

and the residues as

$$
\begin{aligned}
& R_{s}=\frac{-1}{2 \Psi \bar{\omega}}\left(\frac{\omega^{2} \psi_{\ell}}{1+\psi_{\ell}{ }^{2}} \hat{y}(-\bar{\omega})+\frac{\psi_{\ell}-\psi_{1}^{\prime}(0) / \psi_{0}^{\prime}(0)}{1+\psi_{\ell}{ }^{2}} \hat{\ddot{y}}(-\bar{\omega})+\Psi^{2}(-\bar{\omega} \xi(0)+\dot{\xi}(0))\right) \\
& R_{u}=\frac{1}{2 \Psi \bar{\omega}}\left(\frac{\omega^{2} \psi_{\ell}}{1+\psi_{\ell}^{2}} \hat{y}(\bar{\omega})+\frac{\psi_{\ell}-\psi_{1}^{\prime}(0) / \psi_{0}^{\prime}(0)}{1+\psi_{\ell}{ }^{2}} \hat{y}(\bar{\omega})+\Psi^{2}(\bar{\omega} \xi(0)+\dot{\xi}(0))\right) .
\end{aligned}
$$

To set both residues to zero the unique choice of initial conditions is

$$
\begin{aligned}
& \xi(0)=\frac{-1}{2 \Psi \bar{\omega}\left(1+\psi_{\ell}{ }^{2}\right)}\left[\omega^{2} \psi_{\ell}(\hat{y}(\bar{\omega})-\hat{y}(-\bar{\omega}))+\left(\psi_{\ell}-\psi_{1}^{\prime}(0) / \psi_{0}^{\prime}(0)\right)(\hat{\ddot{y}}(\bar{\omega})-\hat{\ddot{y}}(-\bar{\omega}))\right] \\
& \dot{\xi}(0)=\frac{-1}{2 \Psi\left(1+\psi_{\ell}{ }^{2}\right)}\left[\omega^{2} \psi_{\ell}(\hat{y}(\bar{\omega})+\hat{y}(-\bar{\omega}))+\left(\psi_{\ell}-\psi_{1}^{\prime}(0) / \psi_{0}^{\prime}(0)\right)(\hat{\ddot{y}}(\bar{\omega})+\hat{\ddot{y}}(-\bar{\omega}))\right]
\end{aligned}
$$

which, for the sinusoidal reference trajectory, become

$$
\xi(0)=0, \quad \dot{\xi}(0)=\frac{-\omega_{r}\left(\omega^{2} \psi_{\ell}-\left(\psi_{\ell}-\psi_{1}^{\prime}(0) / \psi_{0}^{\prime}(0)\right) \omega_{r}^{2}\right)}{\Psi^{2}\left(1+\psi_{\ell}^{2}\right)\left(\check{\omega}^{2}+\omega_{r}^{2}\right)} .
$$


Note that if only the unstable residue is set to zero one only requires that

$$
\frac{\bar{\Phi}}{\Psi} \xi(0)+\dot{\xi}(0)=\frac{-\omega_{r}}{\Psi^{2}\left(1+\psi_{\ell}{ }^{2}\right)\left(\bar{\omega}^{2}+\omega_{r}{ }^{2}\right)}\left[\omega^{2} \psi_{\ell}-\left(\psi_{\ell}-\psi_{1}^{\prime}(0) / \psi_{0}^{\prime}(0)\right) \omega_{r}^{2}\right]
$$

For this case, a natural question is how should the additional degree of freedom be used. A reasonable requirement would be that the initial flexible variables, $\delta(0)$ and $\dot{\delta}(0)$, are set to zero. This cannot be achieved, as it would lead to a contradiction.

To see how this solution relates to the regulator approach, note that

$$
q(t)=C^{+} y(t)+\tilde{C} \xi(t)
$$

Therefore,

$$
\begin{array}{ll}
\theta_{\text {des }}(0)=0, & \delta_{\text {des }}(0)=0, \\
\dot{\theta}_{\text {des }}(0)=\frac{\dot{y}(0)}{1+\psi_{\ell}{ }^{2}}-\psi_{\ell} \dot{\xi}(0), & \dot{\delta}_{\text {des }}(0)=\frac{\psi_{\ell} \dot{y}(0)}{1+\psi_{\ell}{ }^{2}}+\dot{\xi}(0)
\end{array}
$$

which, after some simple manipulations, can be shown corresponding to the initial conditions in the regulator approach, $P w(0)$.

\section{Time domain method}

The state space representation of system (115) is

$$
\left[\begin{array}{l}
\dot{\xi} \\
\dot{\xi}
\end{array}\right]=\left[\begin{array}{cc}
0 & 1 \\
\omega^{2} & 0
\end{array}\right]\left[\begin{array}{l}
\xi \\
\dot{\xi}
\end{array}\right]+\left[\begin{array}{l}
0 \\
L
\end{array}\right] y(t)
$$

where $L$ is defined as

$$
L=\frac{-\omega^{2} \psi_{\ell}+\omega_{r}^{2}\left(\psi_{\ell}-\psi_{1}^{\prime}(0) / \psi_{0}^{\prime}(0)\right)}{\left(1+\psi_{\ell} \psi_{1}^{\prime}(0) / \psi_{0}^{\prime}(0)\right)\left(1+\psi_{\ell}^{2}\right)}
$$

The eigenvectors associated to the stable and unstable eigenvalues are

$$
v_{s}=\left[\begin{array}{c}
-1 \\
\bar{\omega}
\end{array}\right], \quad v_{u}=\left[\begin{array}{l}
1 \\
\bar{\omega}
\end{array}\right]
$$

so that the following diagonalizing change of coordinates can be done

$$
\left[\begin{array}{l}
\gamma_{+} \\
\gamma_{-}
\end{array}\right]=\frac{1}{2 \bar{\omega}}\left[\begin{array}{cc}
\bar{\omega} & 1 \\
-\bar{\omega} & 1
\end{array}\right]^{-1}\left[\begin{array}{l}
\xi \\
\dot{\xi}
\end{array}\right]
$$

In this new set of coordinates one has

$$
\left[\begin{array}{l}
\dot{\gamma}_{+} \\
\dot{\gamma}_{-}
\end{array}\right]=\left[\begin{array}{cc}
\bar{\omega} & 0 \\
0 & -\bar{\omega}
\end{array}\right]\left[\begin{array}{l}
\gamma_{+} \\
\gamma_{-}
\end{array}\right]+\frac{L}{2 \bar{\omega}}\left[\begin{array}{l}
1 \\
1
\end{array}\right] y(t)
$$

The initial condition $\gamma_{+}(0)$ is chosen to be

$$
\gamma_{+}(0)=\frac{-L}{2 \bar{\omega}} \int_{0}^{\infty} e^{-\bar{\omega} \tau} y(\tau) d \tau=\frac{-L \dot{\omega}_{r}}{2 \bar{\omega}\left(\bar{\omega}^{2}+\dot{\omega}_{\tau}^{2}\right)}=\frac{\dot{\xi}(0)}{2 \bar{\omega}}
$$


while $\gamma_{-}(0)$ is given by

$$
\gamma_{-}(0)=-\int_{-\infty}^{0} e^{\bar{\omega} \tau} \frac{L}{2 \bar{\omega}} y(\tau) d \tau=\frac{\dot{\xi}(0)}{2 \bar{\omega}} .
$$

Given these initial conditions, one can compute the time evolution and obtain

$$
\begin{aligned}
& \gamma_{+}(t)=\frac{-L e^{\bar{\omega} t}}{2 \bar{\omega}} \int_{t}^{\infty} e^{-\bar{\omega} \tau} y(\tau) d \tau=\frac{-L\left(\bar{\omega} \sin \omega_{r} t+\omega_{r} \cos \omega_{r} t\right)}{2 \bar{\omega}\left(\bar{\omega}^{2}+\omega_{r}^{2}\right)} \\
& \gamma_{-}(t)=e^{-\bar{\omega} t}\left[\gamma_{-}(0)+\frac{L}{2 \bar{\omega}} \int_{0}^{t} e^{\bar{\omega} \tau} y(\tau) d \tau\right]=\frac{L\left(\bar{\omega} \sin \omega_{r} t-\omega_{r} \cos \omega_{r} t\right)}{2 \bar{\omega}\left(\bar{\omega}^{2}+\omega_{r}^{2}\right)} .
\end{aligned}
$$

Using the transformation $T$ to compute $(\xi, \dot{\xi}), q(t)$ can be shown to be the same as the regulator solution

$$
\begin{aligned}
& \theta_{\text {des }}(t)=\frac{1}{1+\psi_{\ell}{ }^{2}} y(t)-\psi_{\ell} \dot{\xi}(t)=P_{11} y(t) \\
& \delta_{\text {des }}(t)=\frac{\psi_{\ell}}{1+\psi_{\ell}{ }^{2}} y(t)+\xi(t)=P_{21} y(t)
\end{aligned}
$$

The feedforward can be directly computed from the desired state:

$$
\begin{aligned}
u_{f f}(t) & =\left(B^{T} B\right)^{-1} B^{T}\left(q_{\overline{d e s}}(t)+\Omega^{2} q_{d e s}(t)\right) \\
& =\frac{1}{\psi_{0}^{\prime 2}(0)+\psi_{1}^{\prime 2}(0)}\left[\bar{\theta}_{\text {des }} \psi_{0}^{\prime}(0)+\psi_{0}\left(\bar{\delta}_{\text {des }}+\omega^{2} \delta_{\text {des }}\right)\right] y_{\text {des }}(t)=F_{1} y_{\text {des }}(t)
\end{aligned}
$$

which is again the feedforward from the regulator approach. 